\title{
Chinese expert consensus on conversion therapy for hepatocellular carcinoma (2021 edition)
}

\begin{abstract}
Hui-Chuan Sun ${ }^{1}$, Jian Zhou ${ }^{1}$, Zheng Wang ${ }^{1}$, Xiufeng Liu ${ }^{2}$, Qing Xie ${ }^{3}$, Weidong Jia ${ }^{4}$, Ming Zhao ${ }^{5}$, Xinyu $\mathrm{Bi}^{6}$, Gong $\mathrm{Li}^{7}$, Xueli Bai ${ }^{8}$, Yuan Ji ${ }^{9}$, Li Xu ${ }^{10}$, Xiao-Dong Zhu ${ }^{1}$, Dousheng Bai ${ }^{11}$, Yajin Chen ${ }^{12}$, Yongjun Chen $^{13}$, Chaoliu Dai ${ }^{14}$, Rongping Guo ${ }^{15}$, Wenzhi Guo ${ }^{16}$, Chunyi Hao ${ }^{17}$, Tao Huang ${ }^{18}$, Zhiyong Huang ${ }^{19}$, Deyu $\mathrm{Li}^{20}$, Gang $\mathrm{Li}^{21}$, Tao $\mathrm{Li}^{22}$, Xiangcheng $\mathrm{Li}^{23}$, Guangming $\mathrm{Li}^{24}$, Xiao Liang ${ }^{25}$, Jingfeng $\mathrm{Liu}^{26}$, Fubao Liu ${ }^{27}$, Shichun $\mathrm{Lu}^{28}$, Zheng $\mathrm{Lu}^{29}$, Weifu $\mathrm{Lv}^{30}$, Yilei Mao ${ }^{31}$, Guoliang Shao ${ }^{32}$, Yinghong Shi ${ }^{1,33}$, Tianqiang Song ${ }^{34}$, Guang Tan $^{35}$, Yunqiang Tang ${ }^{36}$, Kaishan Tao ${ }^{37}$, Chidan Wan ${ }^{38}$, Guangyi Wang $^{39}$, Lu Wang ${ }^{40}$, Shunxiang Wang ${ }^{41}$, Tianfu Wen ${ }^{42}$, Baocai Xing ${ }^{43}$, Bangde Xiang ${ }^{44}$,
\end{abstract} Sheng Yan ${ }^{45}$, Dinghua Yang ${ }^{46}$, Guowen Yin ${ }^{47}$, Tao Yin ${ }^{48}$, Zhenyu Yin ${ }^{49}$, Zhengping Yu ${ }^{50}$, Bixiang Zhang ${ }^{19}$, Jialin Zhang $^{51}$, Shuijun Zhang ${ }^{52}$, Ti Zhang ${ }^{53}$, Yamin Zhang ${ }^{54}$, Yubao Zhang ${ }^{55}$, Aibin Zhang ${ }^{56}$, Haitao Zhao ${ }^{57}$, Ledu Zhou ${ }^{58}$, Wu Zhang ${ }^{59}$, Zhenyu Zhu ${ }^{60}$, Shukui Qin ${ }^{61}$, Feng Shen ${ }^{62}$, Xiujun Cai ${ }^{63}$, Gaojun Teng ${ }^{64}$, Jianqiang Cai ${ }^{65}$, Minshan Chen ${ }^{66}$, Qiang $\mathrm{Li}^{67}$, Lianxin Liu ${ }^{68}$, Weilin Wang ${ }^{69}$, Tingbo Liang $^{70}$, Jiahong Dong ${ }^{71}$, Xiaoping Chen ${ }^{19}$, Xuehao Wang ${ }^{72}$, Shusen Zheng ${ }^{73}$, Jia Fan ${ }^{1}$; Alliance of Liver Cancer Conversion Therapy, Committee of Liver Cancer of the Chinese Anti-Cancer Association

\begin{abstract}
${ }^{1}$ Department of Liver Surgery and Transplantation, Liver Cancer Institute, Zhongshan Hospital, Fudan University, Shanghai, China; ${ }^{2}$ Department of Medical Oncology of PLA Cancer Center, Jinling Hospital, Nanjing, China; ${ }^{3}$ Department of Infectious Disease, Ruijin Hospital, Shanghai Jiao Tong University School of Medicine, Shanghai, China; ${ }^{4}$ Department of Liver Surgery, The First Affiliated Hospital of USTC, Hefei, China; ${ }^{5}$ Minimally Invasive Interventional Division, Sun Yat-sen University Cancer Center, Guangzhou, China; ${ }^{6}$ Department of Hepatobiliary Surgery, National Cancer Center/National Clinical Research Center for Cancer/Cancer Hospital, Chinese Academy of Medical Sciences and Peking Union Medical College, Beijing, China; ${ }^{7}$ Department of Radiation Oncology, Beijing Tsinghua Changgung Hospital, Beijing, China; ${ }^{8}$ Department of Hepatobiliary and Pancreatic Surgery, the First Affiliated Hospital, School of Medicine, Zhejiang University, Hangzhou, China; ${ }^{9}$ Department of Pathology, Fudan University Shanghai Cancer Centre, Shanghai, China; ${ }^{10}$ Department of Liver Surgery, Sun Yat-sen University Cancer Centre, Guangzhou, China; ${ }^{11}$ Department of Hepatobiliary Surgery, Clinical Medical College, Yangzhou University, Yangzhou, China; ${ }^{12}$ Department of Hepatobiliopancreatic Surgery, Sun Yat-sen Memorial Hospital, Sun Yat-sen University, Guangzhou, China; ${ }^{13}$ Division of Hepatobiliary Surgery, Department of General Surgery, Ruijin Hospital, Shanghai Jiaotong University School of Medicine, Shanghai, China; ${ }^{14}$ Department of Hepatobiliary and Splenic Surgery, Shengjing Hospital Affiliated to China Medical University, Shenyang, China; ${ }^{15}$ The Department of Hepatobiliary Oncology of Sun Yat-sen University Cancer Center, State Key Laboratory of Oncology in South China, Collaborative Innovation Center for Cancer Medicine, Guangzhou, China; ${ }^{16}$ Department of Hepatobiliary and Pancreatic Surgery, The First Affiliated Hospital of Zhengzhou University, Zhengzhou, China; ${ }^{17}$ Key Laboratory of Carcinogenesis and Translational Research (Ministry of Education), Sarcoma Center, Peking University Cancer Hospital and Institute, Beijing, China; ${ }^{18}$ Department of Hepatobiliary Surgery, Affiliated Tumour Hospital of Zhengzhou University, Zhengzhou, China; ${ }^{19}$ Hepatic Surgery Center, Tongji Hospital, Tongji Medical College, Huazhong University of Science and Technology, Wuhan, China; ${ }^{20}$ Department of Hepato-Biliary Pancreatic Surgery, Henan Provincial People's Hospital, Zhengzhou, China; ${ }^{21}$ Department of Hepatobiliary Pancreatic Surgery, Changhai Hospital, Naval Military Medical University (Second Military Medical University), Shanghai, China; ${ }^{22}$ Department of general surgery, Qilu Hospital, Shandong University, Jinan, China; ${ }^{23}$ Department of Liver Transplantation Center, The First Affiliated Hospital of Nanjing Medical University, Nanjing, China; ${ }^{24}$ Center of General Surgery, Beijing YouAn Hospital, Capital Medical University, Beijing, China; ${ }^{25}$ Department of General Surgery, Zhejiang University, School of Medicine, Sir Run Run Shaw Hospital, Hangzhou, China; ${ }^{26}$ The United Innovation of Mengchao Hepatobiliary Technology Key Laboratory of Fujian Province, Mengchao Hepatobiliary Hospital of Fujian Medical University, Fuzhou, China; ${ }^{27}$ Division of General Surgery, First Affiliated Hospital, Anhui Medical University, Hefei, China; ${ }^{28}$ Department of Hepatobiliary Surgery, First Medical Center of Chinese People's Liberation Army (PLA) General Hospital, Beijing, China; ${ }^{29}$ Department of Hepatobiliary Surgery, The First Affiliated Hospital of Bengbu Medical College Bengbu, China; ${ }^{30}$ Department of Interventional Radiology, The Anhui Provincial Hospital, Hefei, China; ${ }^{31}$ Department of Liver Surgery, Peking Union Medical College (PUMC) Hospital, PUMC \& Chinese Academy of Medical Sciences (CAMS), Beijing, China; ${ }^{32}$ Department of Intervention, Zhejiang Cancer Hospital, Hangzhou, China; ${ }^{33}$ Key Laboratory of Carcinogenesis and Cancer Invasion of Ministry of Education, Shanghai, China; ${ }^{34}$ Department of Hepatobiliary Cancer, Tianjin Medical University Cancer Institute and Hospital, National Clinical
\end{abstract}


Research Center for Cancer, Key Laboratory of Cancer Prevention and Therapy, Tianjin, Tianjin's Clinical Research Center for Cancer, Tianjin, China; ${ }^{35}$ Department of Hepatobiliary Surgery, First Affiliated Hospital of Dalian Medical University, Dalian, China; ${ }^{36}$ Department of HepaticBiliary Surgery, The Affiliated Cancer Hospital of Guangzhou Medical University, Guangzhou, China; ${ }^{37}$ Department of Hepatobiliary Surgery, Xijing Hospital, Fourth Military Medical University, Xi'an, China; ${ }^{38}$ Department of Hepatobiliary Surgery, Union Hospital, Tongji Medical College, Huazhong University of Science and Technology, Wuhan, China; ${ }^{39}$ Department of Hepatobiliary and Pancreatic Surgery, The First Hospital of Jilin University, Changchun, China; ${ }^{40}$ Liver Surgery Department, Shanghai Cancer Center, Fudan University, Shanghai, China; ${ }^{41}$ Department of Hepatobiliary Surgery, The Fourth Hospital of Hebei Medical University, Shijiazhuang, China; ${ }^{42}$ Department of Liver Surgery \& Liver Transplantation Centre, West China Hospital of Sichuan University, Chengdu, China; ${ }^{43}$ Hepatopancreatobiliary Surgery Department I, Key Laboratory of Carcinogenesis and Translational Research, Ministry of Education, Peking University School of Oncology, Beijing Cancer Hospital and Institute, Beijing, China; ${ }^{44}$ Hepatobiliary Surgery Department, Guangxi Liver Cancer Diagnosis and Treatment Engineering and Technology Research Center, Key Laboratory for High-Incidence Tumor Prevention and Treatment, Ministry of Education, Guangxi Medical University Cancer Hospital, Nanning, China; ${ }^{45}$ Division of Hepatobiliary and Pancreatic Surgery, Department of Surgery, The Second Affiliated Hospital, School of Medicine, Zhejiang University, Hangzhou, China; ${ }^{46}$ Unit of Hepatobiliary Surgery, Department of General Surgery, Nanfang Hospital, Southern Medical University, Guangzhou, China; ${ }^{47}$ Department of Intervention, Cancer Hospital of Jiangsu, Nanjing, China; ${ }^{48}$ Department of Hepatic \& Biliary \& Pancreatic Surgery, Hubei Cancer Hospital, Affiliated Hubei Cancer Hospital of Huazhong University of Science and Technology, Wuhan, China; ${ }^{49}$ Department of Hepatobiliary Surgery, Zhongshan Hospital, Xiamen University, Fujian Provincial Key Laboratory of Chronic Liver Disease and Hepatocellular Carcinoma, Xiamen, China; ${ }^{50}$ Department of Hepatobiliary Surgery, The First Affiliated Hospital of Wenzhou Medical University, Wenzhou, China; ${ }^{51}$ Department of Hepatobiliary Surgery, the First Hospital of China Medical University, Shenyang, China; ${ }^{52}$ Key Laboratory of Hepatobiliary and Pancreatic Surgery and Digestive Organ Transplantation of Henan Province, The First Affiliated Hospital of Zhengzhou University, Zhengzhou, China; ${ }^{53}$ Department of Hepatic Surgery, Fudan University Shanghai Cancer Center, Shanghai Medical College, Fudan University, Shanghai, China; ${ }^{54}$ Department of Hepatobiliary Surgery, Tianjin First Central Hospital, Tianjin, China; ${ }^{55}$ Department of Hepatobiliary Pancreatic Surgery, Harbin Medical University Cancer Hospital, Harbin, China; ${ }^{56}$ Department of Hepatobiliary Pancreatic Surgery, The First Affiliated Hospital of College of Medicine, Zhejiang University, Hangzhou, China; ${ }^{57}$ Department of Liver Surgery, Peking Union Medical College Hospital, Chinese Academy of Medical Sciences and Peking Union Medical College, Beijing, China; ${ }^{58}$ Department of Liver Surgery, Xiangya Hospital, Central South University, Changsha, China; ${ }^{59}$ Division of Hepatobiliary and Pancreatic Surgery, Department of Surgery, Key Laboratory of Combined Multi-Organ Transplantation, Zhejiang Province, First Affiliated Hospital, School of Medicine, Zhejiang University, Hangzhou, China; ${ }^{60}$ Hepatoliliary Surgery Center, 302 Hospital of PLA, Beijing, China; ${ }^{61}$ Qinhuai Medical Area, Eastern Theater General Hospital of PLA China, Nanjing, China; ${ }^{62}$ Department of Hepatic Surgery IV, the Eastern Hepatobiliary Surgery Hospital, Second Military Medical University, Shanghai, China; ${ }^{63}$ Department of General Surgery, Sir Run-Run Shaw Hospital, Zhejiang University, Hangzhou, China; ${ }^{64}$ Center of Interventional Radiology and Vascular Surgery, Department of Radiology, Zhongda Hospital, Medical School, Southeast University, Nanjing, China; ${ }^{65}$ Department of Hepatobiliary Surgery, National Cancer Center/National Clinical Research Center for Cancer/Cancer Hospital, Chinese Academy of Medical Sciences and Peking Union Medical College, Beijing, China; ${ }^{66}$ Department of Liver Surgery, Sun Yat-sen University Cancer Center, Guangzhou, China; ${ }^{67}$ Department of Hepatobiliary Surgery, Tianjin Medical University Cancer Institute and Hospital, National Clinical Research Center for Cancer, Key Laboratory of Cancer Prevention and Therapy, Tianjin's Clinical Research Center for Cancer, Tianjin, China; ${ }^{68}$ Department of Hepatobiliary Surgery, Anhui Province Key Laboratory of Hepatopancreatobiliary Surgery, The First Affiliated Hospital of USTC, Division of Life Sciences and Medicine, University of Science and Technology of China, Hefei, China; ${ }^{69}$ Department of Hepatobiliary and Pancreatic Surgery, The Second Affiliated Hospital, Zhejiang University School of Medicine, Hangzhou, China; ${ }^{70}$ Department of Hepatobiliary and Pancreatic Surgery, the Second Affiliated Hospital, Zhejiang University School of Medicine, Hangzhou, China; ${ }^{71}$ Hepatopancreatobiliary Center, Beijing Tsinghua Changgung Hospital, School of Clinical Medicine, Tsinghua University, Changping, Beijing, China; ${ }^{72}$ Hepatobiliary Center, The First Affiliated Hospital of Nanjing Medical University, Key Laboratory of Liver Transplantation, Chinese Academy of Medical Sciences, NHC Key Laboratory of Living Donor Liver Transplantation (Nanjing Medical University), Nanjing, China; ${ }^{73}$ Division of Hepatobiliary and Pancreatic Surgery, Department of Surgery, First Affiliated Hospital, Zhejiang University, School of Medicine, Hangzhou, China

Contributions: (I) Conception and design: All authors; (II) Administrative support: None; (III) Provision of study materials or patients: All authors; (IV) Collection and assembly of data: All authors; (V) Data analysis and interpretation: All authors; (VI) Manuscript writing: All authors; (VII) Final approval of manuscript: All authors.

Correspondence to: Jia Fan. Department of Liver Surgery and Transplantation, Liver Cancer Institute, Zhongshan Hospital, Fudan University, 180 Fenglin Road, Shanghai 200032, China. Email: fan.jia@zs-hospital.sh.cn. 


\begin{abstract}
Recent advances in systemic and locoregional treatments for patients with unresectable or advanced hepatocellular carcinoma (HCC) have resulted in improved response rates. This has provided an opportunity for selected patients with initially unresectable HCC to achieve adequate tumor downstaging to undergo surgical resection, a 'conversion therapy' strategy. However, conversion therapy is a new approach to the treatment of HCC and its practice and treatment protocols are still being developed. Review the evidence for conversion therapy in HCC and develop consensus statements to guide clinical practice. Evidence review: Many research centers in China have accumulated significant experience implementing HCC conversion therapy. Preliminary findings and data have shown that conversion therapy represents an important strategy to maximize the survival of selected patients with intermediate stage to advanced HCC; however, there are still many urgent clinical and scientific challenges for this therapeutic strategy and its related fields. In order to summarize and learn from past experience and review current challenges, the Chinese Expert Consensus on Conversion Therapy for Hepatocellular Carcinoma (2021 Edition) was developed based on a review of preliminary experience and clinical data from Chinese and non-Chinese studies in this field and combined with recommendations for clinical practice. Sixteen consensus statements on the implementation of conversion therapy for HCC were developed. The statements generated in this review are based on a review of clinical evidence and real clinical experience and will help guide future progress in conversion therapy for patients with HCC.
\end{abstract}

Keywords: Hepatocellular carcinoma (HCC); conversion therapy; surgical resection; systematic treatment; locoregional treatment; consensus; China

Submitted Aug 11, 2021. Accepted for publication Nov 18, 2021.

doi: $10.21037 / \mathrm{hbsn}-21-328$

View this article at: https://dx.doi.org/10.21037/hbsn-21-328

\section{Introduction}

Many research centers in China have accumulated significant experience implementing liver cancer conversion therapy. Preliminary findings and data have shown that conversion therapy represents an important strategy to maximize the survival of selected patients with intermediateto advanced-stage liver cancer; however, there are still many urgent clinical and scientific challenges for this therapeutic strategy and its related fields. In order to summarize and learn from past experience and review current challenges, the Chinese Expert Consensus on Conversion Therapy for Hepatocellular Carcinoma (2021 Edition) was developed based on a review of preliminary experience and clinical data from Chinese and non-Chinese studies in this field and combined with recommendations for clinical practice. The consensus is intended for use primarily by doctors involved in the treatment of patients with HCC. We present the following article in accordance with the RIGHT reporting checklist (available at https://hbsn.amegroups.com/article/ view/10.21037/hbsn-21-328/rc.

\section{Methodology}

A group of 80 experts, representing multiple specialties involved in the treatment of HCC implemented a modified Delphi consensus approach to develop guidelines and indications for conversion therapy in HCC. The panelists disclosed potential conflicts of interest and did not receive financial compensation for their participation. Two independent evaluators conducted literature reviews according to the pre-defined search strategy (Tables S1,S2). The literature searches conducted in multiple electronic databases and results exported to Endnote reference managing software. Searches of the PubMed, Web of Science, Cochrane Library, Medline and EMBASE databases were performed in August 2020 and updated in March 2021. No date or study design limits were incorporated into searches in order to return all available evidence, including conference proceedings (although conference proceeding returns were limited to 3 years preceding the date of search). A review of the articles identified during the literature search was undertaken to 
identify and synthesize evidence to support the creation of consensus statements with a supporting narrative synthesis of available evidence.

Two rounds of consensus seminars were held, in Shenzhen on April 23, 2021, and Shanghai on May 9, 2021 respectively. Panelists received literature supporting each draft consensus statements and indicated whether they agreed with each statement. The experts were invited to modify the wording of the recommendations/statements at the 'online-to-offline' virtual meeting in order to come to a consensus on the acceptance or rejection of an adapted recommendation and discuss the applicability and challenges of each recommendation in clinical practice. All statements reached consensus through facilitated discussion and iterative revisions. Panelists were asked to vote using a custombuilt online voting platform (https://www.wjx.cn) to rate their agreement with the statements based on the evidence available, on a scale of $\mathrm{A}$ to $\mathrm{E}$, where $\mathrm{A}=$ accept completely, $\mathrm{B}=$ accept with some reservation, $\mathrm{C}=$ accept with major reservation, $\mathrm{D}=$ reject with some reservation, $\mathrm{E}=$ reject completely. The level of agreement (LoA) was defined as the proportion of panelists who voted A or B. Per Delphi protocol, consensus for each statement was defined as having agreement $(\mathrm{A}+\mathrm{B})$ from at least $80 \%$ of panelists. The discipline and geographical distribution of respondents per statement and recommendation are presented in Table S3.

Following discussion and voting, evidence supporting the statements that achieved $80 \%$ agreement or higher was categorized according to the GRADE system for grading quality of evidence. The quality of evidence scale ranged from 'high' (high confidence in the correlation between true and estimated effect), 'moderate' (moderate confidence in the estimated effect. It is possible that the true effect is very different from the estimated effect), 'low' (limited confidence in the estimated effect. The true effect may be very different from the estimated effect) and 'very low' (very little confidence in the estimated effect. The true effect is very probably different from the estimated effect). The strength of each recommendation was then recorded as 'strong' or 'conditional'. Panelists assigned proposed recommendation strengths to each consensus statement according to the GRADE grid. For a recommendation to be graded as strong rather than conditional, at least $70 \%$ of participants were required to endorse it as strong $(1,2)$. The strength of recommendation was assessed based on considerations of desirable and undesirable anticipated effects, the certainty of the evidence of effects, any important uncertainty about or variability in how much the outcome is valued, whether the balance of these effects favors the intervention or comparison, the acceptability of the intervention to key stakeholders and feasibility of intervention implementation (2). The quality of evidence is only one of the factors that determine the recommendation strength, and low-quality evidence may also lead to a strong recommendation.

\section{Current status and challenges in the treatment of liver cancer}

Primary liver cancer is one of the most common malignant tumors in the world $(3,4)$, ranking $6^{\text {th }}$ in the global incidence of malignant tumors in 2018 , and $4^{\text {th }}$ among the leading causes of tumor death (5). In China, the incidence of primary liver cancer ranked $4^{\text {th }}$ among malignant tumors in 2015, and $3^{\text {rd }}$ among the leading causes of tumor death (6). Primary liver cancer includes hepatocellular carcinoma (HCC) (comprising $75-85 \%$ of cases) and intrahepatic cholangiocarcinoma (comprising $10-15 \%$ of cases) as well as other rare types, and this consensus focuses on HCC (3).

While the Barcelona Cancer Liver Clinic (BCLC) staging system for liver cancer is employed extensively throughout the US and Europe, in China the China Liver Cancer Staging (CNLC) system is preferred because of its relevance to local systems and practices $(7,8)$. A comparison of the BCLC staging system and CNLC staging system is shown in Figure 1. Early, and some intermediate, stage HCC (mainly CNLC stage Ia, Ib and some patients with stage IIa) $(4,7)$ are indicated for curative treatment such as surgical resection, local ablation and liver transplantation, and the median survival time can exceed 5 years $(4,7)$. Unfortunately, most Chinese patients with HCC are already in the intermediate or advanced stages (CNLC stage IIb, IIIa, and IIIb, covering a proportion of patients with BCLC stage $B$ and all patients with BCLC stage $C$ ) when they are first diagnosed. According to the BRIDGE study, $64 \%$ of patients with liver cancer in China have CNLC stage II and III disease at the time of first diagnosis (equivalent to BCLC stage $B$ and C) (9), and the associated median survival time with standard treatment is around 1 year (10) and is even shorter in Chinese patients $(4,7,11,12)$. For the majority of patients with intermediate and advanced stage HCC, nonsurgical local or systemic treatment are the predominant treatment choices. Despite this, a small proportion of carefully selected patients with intermediate or advanced stage HCC are able to undergo surgical resection, and the clinical efficacy of surgical excision may be better than 


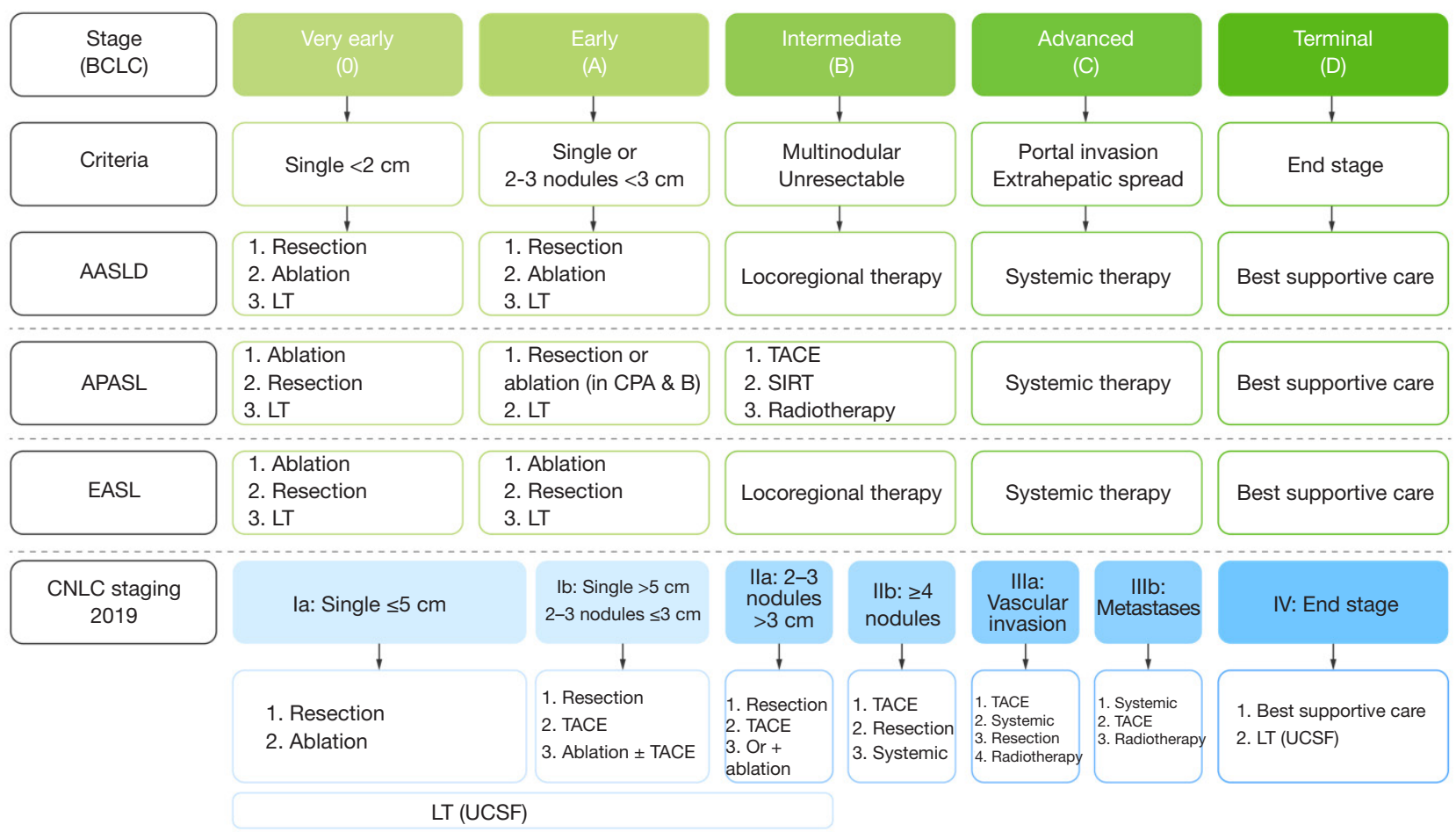

Figure 1 Summary of stage-dependent recommendations on the treatment of HCC by the international guidelines and Chinese guideline. AASLD, American Association for the Study of Liver Disease; APASL, Asian Pacific Association for the Study of the Liver; CNLC, China Liver Cancer Staging; BCLC, Barcelona Clinic Liver Cancer; CPA \& B, Child-Pugh class A and B; EASL, European Association for the Study of the Liver; LT, liver transplantation; UCSF, University of California San Francisco; SIRT, selective internal radiation therapy; TACE, transarterial chemoembolization; HCC, hepatocellular carcinoma.

that of non-surgical treatment (13). However, among these patients, the short-term recurrence rate after surgery is very high $(14,15)$, and surgery fails to provide a curative outcome for most of these patients.

In recent years, significant progress has been made in the non-surgical treatment of HCC. Systemic therapy for advanced or unresectable HCC, especially anti-angiogenic drugs combined with immunotherapy, can achieve an objective response rate (ORR) of about $30 \%$, and the median survival time for patients receiving this type of therapy can be as long as 20 months (16-19). There are a variety of such targeted anti-cancer drugs available in China, and the associated treatment cost has dropped significantly in recent years, with certain drugs now covered/partially covered by national medical insurance. It is expected that this type of combination treatment with tyrosine kinase inhibitors (TKIs) and immunotherapy will soon be widely used in advanced HCC in China. In addition, local treatments such as transcatheter arterial chemoembolization (TACE), hepatic artery infusion chemotherapy (HAIC) $(20,21)$ and radiotherapy (22) combined with other therapeutic measures through the improvement of technology and drugs, have not only achieved better results than before in shrinking tumors and tumorous thrombus, but have also improved patients' survival.

\section{History, patient population and definition of conversion therapy of HCC}

The history of conversion therapy in HCC can be traced back to the 1970s when a case report described removing a giant hepatoblastoma after shrinking the tumor through chemotherapy and radiotherapy (23). In the 1990s, many centers reported a series of studies on tumor shrinkage, downstaging, and resection after TACE or internal radionuclide radiotherapy and external radiotherapy (24-28); more importantly, these studies reported that the 5 -year survival rate for patients receiving a 'conversion and 
resection' can reach $50-60 \%$, which is equivalent to the survival rate after early liver cancer excision $(24,29,30)$. The China National Health Commission's "Guidelines for the Diagnosis and Treatment of Hepatocellular Carcinoma (2019 Edition)" lists conversion therapy as one of the treatments for unresectable HCC (7).

Conversion therapy is referring to conversion of an unresectable HCC into resectable HCC followed by surgical removal of the tumor. Therefore, how to define "unresectable HCC" is a key question. Unresectable HCC can be divided into two categories. One is unresectable in the sense of surgery, including the patient's inability to withstand surgical trauma in terms of their general condition, liver function, and insufficient remaining liver volume (surgically unresectable). The second category of unresectable liver cancer may be technically resectable, but after resection, no better efficacy can be obtained compared with non-surgical treatment (oncologically/biologically unresectable). The definition of surgically unresectable HCC is widely agreed, but for oncologically/biologically unresectable disease the definition is dynamic and more controversial. Overall, a long-term benefit in overall survival is more important than a successful resection.

In the past, when there was a lack of effective non-surgical local and drug treatments, even for advanced HCC, if the disease was surgically resectable, the postoperative survival would be better than that with non-surgical treatment in selected patients $(13,31,32)$. At that time, unresectable HCC was defined as unresectable in the sense of surgery. Accordingly, the main goal of conversion therapy is to eliminate the factors contributing to HCC being considered unresectable in the sense of surgery. It is important to note that there is overlap between conversion therapy and neoadjuvant therapy in patients with surgically resectable tumors. Conversion therapy includes the conversion of unresectable cancers to resectable cancers in the surgical sense, as well as the conversion of patients with poor predicted outcomes after resection (CNLC-Stages IIb and IIIa) to those with better outcomes after resection (i.e., conversion in the oncological sense). In contrast, neoadjuvant therapy is generally a preoperative treatment for patients with resectable cancer that aims to improve their oncological outcomes (including quality of survival and long-term survival prognosis). Therefore, the ultimate goal of conversion and neoadjuvant therapies is the same for patients with locally advanced HCC that is resectable in the surgical sense.

At present, the median overall survival of patients with unresectable advanced HCC after receiving systemic drug treatment is around 20 months $(16,17)$. For HCC characterized by resectable intrahepatic lesions and concurrent vascular invasion (technically resectable, CNLC stage IIIa) the median postoperative survival time is $12-15$ months (14), which is lower than that for patients with a similar stage disease who receive first-line systemic drug therapy. Obviously, for such patients (CNLC stage IIIa), surgical resection may not be the first choice of treatment. Furthermore, for patients with CNLC stage IIb disease, the median survival time following locoregional therapy has reached about 30 months (33). Although randomized controlled studies have shown patients with multi-nodular tumors receiving surgical resection achieve better survival outcomes versus those receiving TACE, this conclusion is mainly applicable to Bolondi stage B1 and B2 liver cancer $(32,34,35)$. In addition, some studies have reported that for multi-nodular tumors (mostly CNLC stage IIb) that exceed the "up-toseven criteria' (the Kinki criteria), treatment with the TKI lenvatinib leads to a median survival of 37 months (36). Therefore, for patients with CNLC stage IIb disease, the long-term survival following surgical treatment is comparable to that following TACE or even systemic treatment. In the "Chinese Guideline for the Diagnosis and Treatment of Hepatocellular Carcinoma (2019 Edition)", for CNLC stage IIb and IIIa HCC, TACE and systemic treatment are recommended as the first choice, and surgical resection is the second and third treatment option, respectively (7). If the tumor burden can be reduced to increase the R0 resection rate and reduce surgery risk, or the tumor can be resected after downstaging, so that the patient can obtain more survival benefits than with other treatments, the whole treatment can thus be defined as conversion therapy.

HCC can be regarded as "potentially resectable" in two broad situations; either the intrahepatic lesion is surgically resectable stage IIb, IIIa HCC (i.e., limited tumor burden), or surgically unresectable stage Ia, Ib, and IIa HCC (classed as unresectable because the remaining liver volume or the surgical margin is not sufficient). Liver cancers meeting the above two descriptions have a relatively high chance of being successfully converted to resectable disease. Therefore, for these potentially resectable liver cancers, more active conversion strategies can be adopted, including high-intensity, multi-modal combined treatment to achieve tumor shrinkage and downstaging in a short period of time, or increase the size of the remnant liver, and ultimately obtain opportunities for radical resection. For patients not meeting the criteria for potentially resectable disease, appropriate anti-tumor treatments should be implemented, 


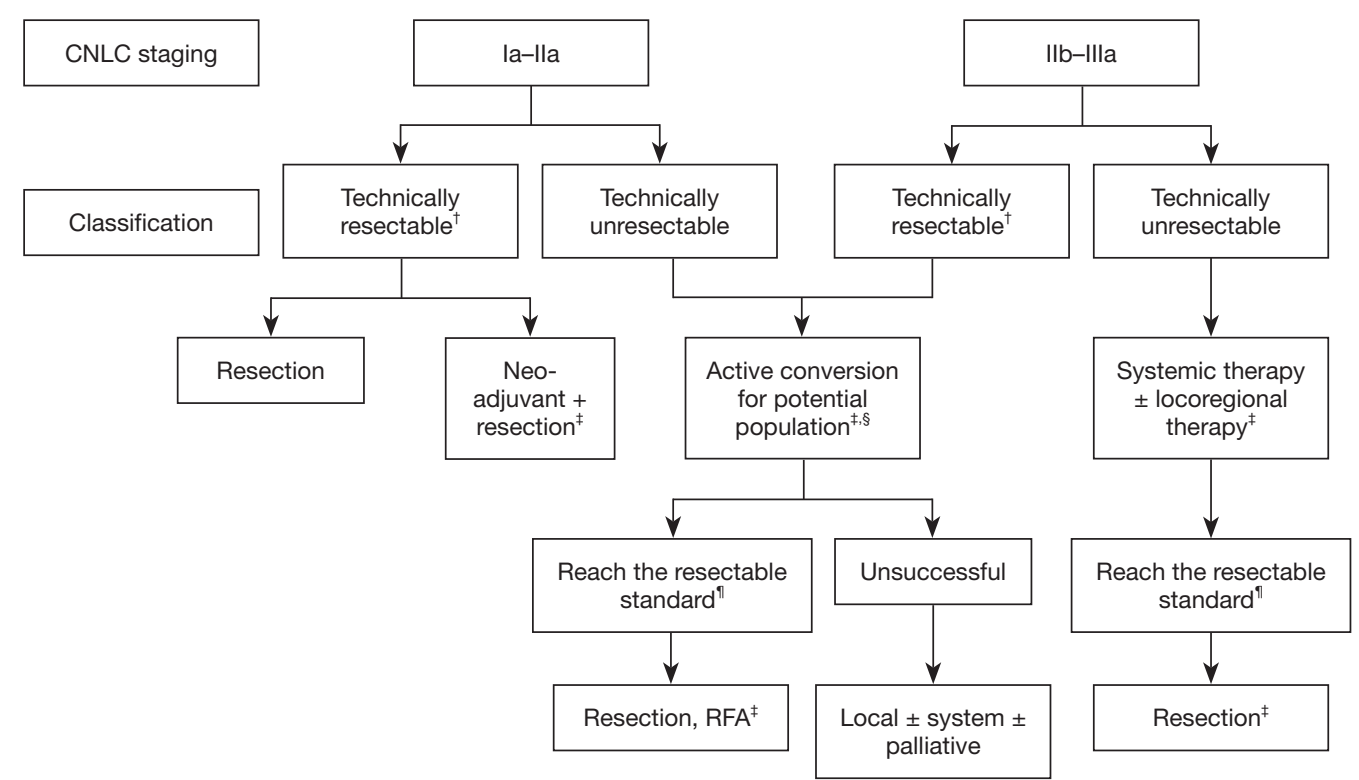

Figure 2 Roadmap of conversion therapy. ${ }^{\dagger}$, technically resectable tumors: R0 resection, adequate FLR, liver function Child-Pugh class A + some Child-Pugh class $\mathrm{B} ;{ }^{\ddagger}$, clinical research; ${ }^{\S}$, include: potent systemic therapy, multimodal therapy (local + systemic), PVE or ALPPS, aggressive hepatoprotective therapy, and combination therapy; " , criteria for oncologically resectable tumors: CNLC Stages IIb-IIIa: tumor down-stagingMVTT necrosis (mRECIST criteria), or partial response, or stable disease lasting 3-4 months; Stages Ia-IIa: adequate FLR, no tumor progression during treatment. FLR, future liver remnant; ALPPS, associating liver partition and portal vein ligation for staged hepatectomy; CNLC, China Liver Cancer Staging; RFA, radiofrequency ablation; MVTT, macrovascular tumor thrombi; PVE, portal vein embolization.

with the patient's quality of life, survival time and treatment costs taken into consideration.

It should be pointed out that conversion and resection is an intermediate goal for the treatment of intermediate or advanced HCC, and long-term survival is the ultimate goal. However, the expected value of a conversion and resection in terms of improving survival is currently based on the known efficacy of resection of early HCC and the results of previous retrospective studies. The expected value of the conversion therapy strategy still requires validation through further clinical research. The value of other treatment methods in patients receiving successful conversion also requires further exploration.

\section{Statement 1}

The definition of unresectable HCC can be divided into surgical causes and oncological causes. Surgical causes refer to the inability to perform safe surgical resection and oncological causes refer to the predicted efficacy after surgery not surpassing other non-surgical treatment methods. The goal of conversion therapy is to eliminate these two causes and achieve a conversion from unresectable to resectable HCC. Surgically unresectable CNLC stage Ia, Ib, IIa HCC and surgically resectable CNLC stage IIb and IIIa HCC are all potentially resectable HCC, and multi-modal, highintensity treatment strategies can be explored and adopted to facilitate the conversion. For surgically unresectable CNLC stage IIb and IIIa HCC, it is recommended that the current treatment norms should be followed, and a sequential treatment strategy should be adopted, with both the intensity and safety of treatment taken into account, and surgical excision conducted when applicable (Figure 2). (Based on expert opinion) (GRADE: strong recommendation, lowquality evidence. Agreement: 96.2\%).

\section{Statement 2}

Resection after tumor shrinkage or downstaging potentially allows patients with intermediate or advanced HCC to achieve long-term survival. Other treatment methods, such as upfront surgical resection, continued medication, or a combination with other local treatment methods, may also enable patients to achieve long-term survival. Controlled 
studies are needed to verify the pros and cons of these different treatment strategies. Extending patients' survival is the ultimate goal of conversion therapy. (Evidence-based) (GRADE: strong recommendation, low-quality evidence. Agreement: 98.1\%).

\section{Significance of formulating an expert consensus on conversion therapy}

Conversion therapy for unresectable HCC has been explored many years ago, and successful cases are not rare; there have been multiple reports of successful conversion from small-sample retrospective cohort studies (20,21,37-40). However, there remain many controversies and questions. For example, what is the role of currently available therapies as part of a conversion strategy? How important is consideration of underlying liver function, and how should treatments of the liver parenchyma be implemented in conversion therapy? Does the tumor need to be surgically resected after tumor response? How should the optimal timing of surgical resection be decided? Is it safe for patients with successfully converted tumors to undergo surgical resection? Is adjuvant treatment needed after resection? How should patients who have failed conversion therapy be managed? How should treatment be tailored for patients with extrahepatic metastasis? Answering these questions and resolving disputes requires not only the experience of experts, but also clarification, concentration, and cooperative studies. Building a consensus is the first step and the basis for future research.

\section{Methods of conversion therapy}

\section{Conversion therapy for tumors}

\section{Systemic (drug) treatment}

At present, the research on conversion therapy mostly comprises small-sample retrospective clinical studies. In China, Zhang et al. reported 35 cases of patients with CNLC stage IIIa HCC treated with programmed death-1 (PD-1) inhibitors combined with TKIs, and the conversion resection rate was $42.4 \%$ (40); Zhu et al. reported 63 cases of patients with initially unresectable liver cancer treated with PD-1 inhibitors combined with TKIs, and the conversion resection rate was $15.9 \%$ (39). In clinical practice, the optimal choice of systemic treatment for patients with potentially resectable HCC is still unclear. Clinical data for systemic therapy in the first-line setting show that lenvatinib has a higher ORR [24.1\%, including $2 \%$ of complete response (CR)] than sorafenib (10). In addition, targeted therapy combined with immunotherapy, such as lenvatinib combined with pembrolizumab, bevacizumab combined with atezolizumab, bevacizumab analogs combined with sintilimab, and apatinib combined with camrelizumab (16-18,41), are associated with ORRs of over $20 \%$ in the treatment of unresectable liver cancer, and therefore has a stronger conversion potential than monotherapy. In addition to anti-tumor effect of the regime, its safety and accessibility need to be taken into consideration (Table 1). Currently, there is a lack of comparative studies of these combinations.

From the perspective of achieving a successful conversion, the most important factors to be considered are ORR and patterns of response, including primary tumor progression rate (PD rate), time to response (TTR), duration of response (DoR) and depth of reduction. A lower PD rate indicates that fewer patients have tumor progression during conversion therapy; rapid onset helps to reduce the exposure time to conversion therapy and thus reduces the incidence of adverse reactions; deeper reduction means a higher probability of tumor shrinkage and downgrading, which is undoubtedly more conducive to later treatment and a longer DoR indicates that the duration of tumor response is longer and provides a longer time window for subsequent treatment.

It should also be considered that systemic therapy alone may enable patients to obtain long-term tumor control and achieve long-term survival. The Keynote524 study showed that among 104 patients who received lenvatinib plus pembrolizumab, 36 achieved a tumor response (ORR $36 \%$, by RECIST v1.1), of whom $73 \%$ achieved long-term response (response duration $\geq 6$ months) (16). Furthermore, the GO30140 study showed that among 104 patients who received atezolizumab plus bevacizumab, 37 achieved a tumor response (ORR 36\%, by RECIST v1.1). Among those patients, $62 \%$ achieved deep response (the total reduction of target lesions $\geq 65 \%$ ), $65 \%$ achieved long-term response (response duration $\geq 6$ months), and $54 \%$ achieved both deep response and long-term response (53). Whether surgical resection is still of therapeutic value in patients achieving such a robust response to systemic therapy needs to be confirmed by comparative study. The proportion of long-term responders and the survival time of patients are among the comparison criteria for evaluating the value of excision treatment.

The response of lesions in different organs/locations is heterogeneous when treated by systemic treatment. Huang 
Table 1 Efficacy and safety summary of systemic and local treatments for patients with advanced HCC

\begin{tabular}{|c|c|c|c|c|c|c|c|}
\hline Treatment regimen & Study name/design & $\begin{array}{l}\text { Sample } \\
\text { size }\end{array}$ & $\begin{array}{l}\mathrm{ORR}^{\dagger}, \\
\%\end{array}$ & $\begin{array}{l}\mathrm{PFS}^{\dagger} \\
\text { months }\end{array}$ & $\begin{array}{c}\text { OS, } \\
\text { months }\end{array}$ & $\begin{array}{l}\text { Grade } \geq 3 \\
\text { TRAEs, \% }\end{array}$ & $\begin{array}{l}\text { Treatment } \\
\text { line }\end{array}$ \\
\hline \multicolumn{8}{|l|}{ TKI+ PD-1/PD-L1 monoclonal antibody } \\
\hline Lenvatinib + pembrolizumab (16) & Phase $\mathrm{Ib}$, single arm & 100 & 36 & 8.6 & 22.0 & 67 & First line \\
\hline Apatinib + camrelizumab (18) & Phase II, single arm & 70 & 34 & 5.7 & 20.3 & $77.4^{\Uparrow}$ & First line \\
\hline $\begin{array}{l}\text { Cabozantinib + nivolumab + } \\
\text { ipilimumab (44) }\end{array}$ & $\begin{array}{l}\text { CheckMate 040: Phase I/II } \\
\text { non-randomized }\end{array}$ & 35 & 29 & 6.8 & NR & 71 & $\begin{array}{l}\text { First line/ } \\
\text { second line }\end{array}$ \\
\hline Anlotinib + penpulimab (45) & Phase Ib/ll, single arm & 31 & 24 & - & NE & 12.9 & First line \\
\hline Cabozantinib + nivolumab (44) & $\begin{array}{l}\text { CheckMate 040: Phase I/II } \\
\text { non-randomized }\end{array}$ & 36 & 19 & 5.4 & 21.5 & 47 & $\begin{array}{l}\text { First line/ } \\
\text { second line }\end{array}$ \\
\hline Bevacizumab + toripalimab (46) & CT34: Phase II, multi-center, single arm & 54 & 31.5 & 9.9 & NR & $37^{\S}$ & First line \\
\hline Bevacizumab + atezolizumab (47) & IMbrave150: Phase III, randomized & 336 & 30 & 6.9 & 19.2 & 43 & First line \\
\hline Bevacizumab $^{\dagger \dagger}+$ sintilimab (41) & ORIENT-32: Phase II/III, randomized & 380 & 21 & 4.6 & NR & 35 & First line \\
\hline \multicolumn{8}{|l|}{ Other options } \\
\hline Nivolumab + ipilimumab ${ }^{\ddagger \ddagger}(48)$ & $\begin{array}{l}\text { CheckMate 040: Phase I/II } \\
\text { non-randomized (sub-analysis) }\end{array}$ & 50 & 32 & - & 22.8 & 53 & Second line \\
\hline Camrelizumab + FOLFOX4 (49) & Phase II, single arm & 34 & 29.4 & 7.4 & 11.7 & 85.3 & First line \\
\hline Durvalumab + tremelimumab ${ }^{\S \S}(50)$ & Phase II, randomized & 74 & 24 & 2.17 & 18.7 & 35.1 & Second line \\
\hline DEB-TACE + placebo (52) & Phase III, randomized & 156 & 31 & $7.8^{\dagger+t}$ & $19.9^{t+t}$ & NR & First line \\
\hline
\end{tabular}

Only studies with sample sizes of $>30$ cases, including published articles and conference reports, were included. Direct comparisons between different clinical trials are not appropriate. ${ }^{\dagger}$, evaluated using RECIST v1.1; ${ }^{\ddagger}$, evaluated using mRECIST; ${ }^{\S}$, treatment-emergent

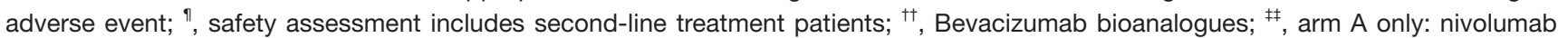
$1 \mathrm{mg} / \mathrm{kg}+$ ipilimumab $3 \mathrm{mg} / \mathrm{kg}$ q3w $\times 4$ followed by nivolumab $240 \mathrm{mg}$ q2w; ${ }^{\S}$, arm T300+D only: tremelimumab 300 mg + durvalumab $1,500 \mathrm{mg} \times 1$ followed by durvalumab $1,500 \mathrm{mg} \mathrm{Q} 4 \mathrm{~W}$; ${ }^{1 \uparrow}$, severe adverse events; ${ }^{\mathrm{t \dagger}}$, days in original/data obtained by conversion. HCC, hepatocellular carcinoma; ORR, objective response rate; PFS, progression free survival; OS, overall survival; TRAE, treatment-related adverse events; TKI, tyrosine kinase inhibitor; PD-1, programmed cell death protein 1; PD-L1, programmed cell death ligand 1; NE, unable to evaluate; NR, not reached; FOLFOX, 5-fluorouracil and oxaliplatin; HAIC, hepatic arterial infusion chemotherapy; TACE, transcatheter arterial chemoembolization; DEB-TACE, drug-eluting bead transarterial chemoembolization.

et al. found that in HCC patients treated with lenvatinib combined with a PD-1 inhibitor, the response rates and duration of response of tumor thrombus were higher than those of intrahepatic lesions. This suggests that the excision/control of intrahepatic lesions is crucial to CNLC stage IIIa HCC, which means that patients with potentially resectable CNLC stage IIIa tumor are more likely to achieve oncological conversion when receiving systemic treatment (54), or combination treatment using systemic therapy and locoregional therapy. 


\section{Statement 3}

Anti-angiogenic drugs or molecular targeted therapy combined with immunotherapies have become an important method for treating unresectable or intermediate and advanced HCC, and for conversion therapy of potentially resectable HCC. The depth, speed and duration of tumor response and organ-specific tumor response are important factors that affect treatment decisions. The safety of medication and its impact on the safety of subsequent operations requires further evaluation. (Evidence-based) (GRADE: strong recommendation, low-quality evidence. Agreement: 100\%).

\section{Locoregional treatment}

\section{Transarterial chemoembolization (TACE)}

Before the advent of effective systemic treatments, TACE was the main method of conversion therapy for unresectable HCC. A single-center randomized controlled trial showed that 21 of 73 patients $(28.8 \%)$ who received liver transplantation exceeded the Milan standard when first examined and received liver transplantation after downstaging through TACE, and overall survival time of patients receiving liver transplantation after conversion therapy is similar to that of patients receiving liver transplantation who were within the Milan standard (55). Several other RCTs also showed that TACE creates potential resection opportunities for patients with initially unresectable HCC and can bring survival benefits (56-58). In 2016, a retrospective study of 831 Chinese patients with HCC found that for the 82 patients who achieved a partial response (PR) after TACE treatment, those who received surgical excision had a longer overall survival (49 vs. 31 months, $\mathrm{P}=0.027$ ) and a higher 5 -year survival rate $(26 \%$ vs. $10 \%)$ compared with those who received conservative treatment (28). Recently, Li et al. reported a retrospective assessing the efficacy of TACE combined with HAIC on initially inoperable patients with HCC, in which $56.1 \%$ of the enrolled patients had tumors $\geq 10 \mathrm{~cm}$ in diameter. The results showed that the conversion rate of TACE combined with HAIC was higher than that of TACE monotherapy (48.8\% vs. 9.5\%; $\mathrm{P}<0.001)$ (59). However, it should also be considered that TACE can have negative effects on liver function or cause liver damage, although these events are rare (60).

\section{Statement 4}

The role of TACE in conversion therapy has been explored and widely recognized. However, it should be noted that multiple TACE procedures may cause liver damage, thereby affecting the safety of liver excision after conversion.
In future, the conversion success rate can be enhanced by improving TACE treatment methods or utilizing combination therapy strategies. (Evidence-based) (GRADE: strong recommendation, moderate-quality evidence. Agreement: 100\%).

\section{Hepatic artery infusion chemotherapy (HAIC)}

In recent years, Chinese scholars have made significant progresses in the field of HAIC treatment of intermediate or advanced HCC. A multi-center RCT showed that the ORR of HAIC treatment among HCC patients with portal vein tumor thrombus was significantly higher than that of sorafenib (mRECIST criteria, $27.6 \%$ vs. 3.4\%, $\mathrm{P}=0.001$ ) (61). A retrospective study published by Lyu et al. also found that the ORR of HAIC treatment was higher than that for sorafenib (mRECIST criteria, $47.8 \%$ vs. $9.1 \%, \mathrm{P}<0.01$ ). $26.1 \%$ of patients in the HAIC treatment group achieved tumor downstaging and had the opportunity to receive local treatment (62). Another RCT study compared the efficacy of combination therapy with HAIC and sorafenib versus sorafenib monotherapy in patients with HCC and portal vein invasion, and the results showed that the overall response rate among the combination therapy group was significantly higher than that of the sorafenib monotherapy group. In addition, $12.8 \%$ of the patients in the combination therapy group achieved downstaging after treatment and received radical surgical resection, of which three patients achieved a pathological complete response (pCR) (20). A further retrospective study also showed that, compared with lenvatinib monotherapy, lenvatinib combined with toripalimab and HAIC can achieve a higher ORR and a higher conversion resection rate $(12.7 \%$ vs. $0 \%)$ (63). These studies suggest that systemic therapy combined with local therapy can achieve higher anti-tumor activity, thereby allowing more patients the possibility of receiving resection. A study comparing the efficacy of HAIC and TACE in patients with BCLC stage B HCC with the largest tumor $>7 \mathrm{~cm}$ in diameter showed that the proportion of patients in the HAIC group who received surgical resection was significantly higher than that in the TACE group (23.9\% vs. $11.5 \%, \mathrm{P}=0.004)$ (51). However, this result may be limited to patients with large tumors, diffuse HCC, and HCC with portal vein tumor thrombus. In other HCC patients with lighter tumor burden, TACE may still have better efficacy (64).

\section{Statement 5}

For patients with HCC whose tumor burden is concentrated in the liver or those with portal vein tumor thrombus, multiple clinical studies have confirmed that HAIC 
treatment has a higher tumor response rate than TACE or systemic therapies. After HAIC treatment, some patients achieve significant tumor burden reduction or large vessel tumor thrombus regression, thus obtaining the opportunity for surgical resection or ablation treatment. HAIC treatment is suitable for patients with tumor burden in the liver and relatively good liver function (Child-Pugh $\mathrm{A} / \mathrm{B}$ ), and the FOLFOX regimen is recommended for chemotherapy. Generally, it is necessary to complete 4 or more consecutive infusion courses to obtain the best chance of conversion. Targeted therapy and immunotherapy combined with HAIC have a higher conversion rate than HAIC alone. (Evidencebased) (GRADE: conditional recommendation, moderatequality evidence. Agreement: $88.5 \%$ ).

\section{Selective internal radiation therapy (SIRT)}

SIRT is also called transcatheter arterial radioembolization (TARE). So far, there have been no large-scale prospective RCTs of SIRT as conversion therapy for patients with intermediate or advanced HCC. A study published by Lau et al. that included 71 patients with unresectable HCC showed that $26.7 \%$ of the patients had tumor shrinkage greater than $50 \%$ after SIRT treatment, of which four patients $(5.6 \%)$ received radical resection and two $(2.8 \%)$ achieved a pCR (65). In a subsequent study, the same group observed 49 patients with advanced HCC who were successfully downstaged after chemotherapy or SIRT and surgically resected, the results suggested that the 5-year survival rate was as high as $57 \%(25)$. Another clinical study reported that the PR rate associated with SIRT in HCC patients with Child Pugh class A liver function and portal vein tumor thrombus was $40 \%$, and in those with Child Pugh class B the PR rate was 25\% (66). An RCT comparing the efficacy of TACE and SIRT in the treatment of unresectable HCC showed that SIRT led to a higher ORR than TACE $(30.8 \%$ vs. $13.3 \%, \mathrm{P}<0.05)$, and $15.4 \%$ of patients in the SIRT group achieved tumor downstaging (38).

\section{Statement 6}

Multiple studies have suggested that SIRT effectively causes tumor shrinkage and has a role in conversion therapy. For patients with portal vein tumor thrombus, SIRT has a higher local dose and a more precise delivery than external beam radiotherapy, and it also reduces radiation damage to normal liver tissue (67). However, clinical data for SIRT in China are currently limited and more evidence is needed to verify its role in conversion therapy. (Evidencebased) (GRADE: conditional recommendation, low-quality evidence. Agreement: 96.2\%).

\section{Radiotherapy}

Japanese researchers compared the efficacy of radiotherapy followed by surgery and upfront surgery in a group of patients with tumor thrombus in the main portal vein or the major branches. Radiotherapy was only targeted to the tumor thrombus, and the radiation dose was 30-36 Gy/10-12 times. Surgery was performed within 2 weeks after radiotherapy. Post-operation pathology showed that in the radiotherapy and resection group, 5/6 (83.3\%) of patients achieved complete pathological necrosis of the main portal vein tumor thrombus. The 5 -year survival rate of the radiotherapy and resection group was $34.8 \%$, and that of the upfront surgery group was $13.1 \%(\mathrm{P}=0.0359)$ (68). For patients with technically resectable CNLC stage IIIa HCC, an RCT reported by Wei et al. compared the efficacy of preoperative radiotherapy followed by resection and upfont resection and found that $20.7 \%$ of patients in the radiotherapy group had portal vein tumor thrombus downstaging from Cheng's type III to Type II or from type II to type I (69). Preoperative radiotherapy followed by resection significantly improved the survival of patients compared with upfront resection (69).

Data also suggest that radiotherapy combined with HAIC may lead to a higher conversion rate. A retrospective study investigated radiotherapy combined with HAIC in the treatment of locally advanced and initially unresectable HCC. A total of 41 patients in this study (16.9\%) underwent surgery after tumor downstaging. The 5 -year OS rate of the surgical group and the non-surgery group was $49.6 \%$ and $9.8 \%(\mathrm{P}<0.001)$ respectively $(70)$. Another retrospective study showed that in patients with BCLC stage C HCC who received combination therapy with radiotherapy and HAIC, the surgical conversion rate was $10.7 \%(68 / 637)$. The median survival time of surgical and non-surgical patients was 103.8 vs. 11.4 months $(\mathrm{P}<0.001)$ and patients receiving a radiotherapy dose $>72$ Gy had higher conversion rates (71).

\section{Statement 7}

For patients with HCC and portal vein tumor thrombus, surgery can be conducted after downstaging through radiotherapy combined with HAIC. Radiotherapy combined with HAIC therapy may achieve a higher conversion rate than either therapy alone. (Evidence-based) (GRADE: conditional recommendation, low-quality evidence. Agreement: 80\%).

\section{Treatment for liver parenchyma}

Increasing the volume of the remaining liver

It has been well established that a safe hepatectomy should 
be conducted in patients with normal liver function [ChildPugh A, indocyanine green retention rate at $15 \mathrm{~min}$ (ICG-R15) $<10 \%$ ], and sufficient future liver remnant (FLR) /standard liver volume (SLV) should be $>20-30 \%$ in non-cirrhotic patients, and $>40 \%$ in those with chronic liver disease or liver parenchymal damage (including liver cirrhosis, severe fatty liver and chemotherapy-related liver damage) (7,72-76). Patients with liver damage need to retain more FLR (e.g., ICG-R15 =10-20\%, patients with chronic liver disease and cirrhosis must have FLR/SLV >50\%) $(77,78)$. Insufficient FLR is an important criterion for determining unresectable liver cancer. For these patients, the goal of conversion therapy is to change from insufficient FLR to sufficient FLR.

Portal vein embolization (PVE) has been in clinical use for a long time, with a conversion rate of $60-80 \%$, and a complication rate of $10-20 \%$ (79-81). The hyperplasia of the remaining liver parenchyma after PVE takes a relatively long time (usually 4-6 weeks). In addition, more than $20 \%$ of patients lose the chance of surgery due to tumor progression or insufficient remaining liver hyperplasia $(74,80,82,83)$. For these patients, the current treatment strategies include combinations of TACE (84), hepatic vein embolization (HVE) $(80,85)$, associating portal embolization and artery ligation (APEAL) (86) and associating liver partition and portal vein ligation for staged hepatectomy (ALPPS) to achieve tumor removal $(84,87)$, to further promote FLR hyperplasia and control tumor progression.

Contraindications for PVE include tumor thrombus of $\mathrm{Vp} 3-\mathrm{Vp} 4$ type, extensive tumor metastasis, severe portal hypertension and coagulation dysfunction (88). For patients expected to have a relatively long FLR hyperplasia time (for example, patients with relatively severe liver cirrhosis, senile patients), and patients with rapid tumor progression, PVE should be used with caution.

ALPPS can usually induce a hyperplasia rate of $47-192 \%$ of the remaining liver in around 1 to 2 weeks, which is much higher than PVE. In addition, due to the short interval between two operations, the risk of tumor progression before resection can be minimized, and the associated tumor resection rate is $95-100 \%$ (89-93). An RCT conducted by Li et al. showed that, compared with PVE, TACE combined with ALPPS has a higher conversion excision efficiency and long-term survival rate, but it also has a higher risk of perioperative complications (94). The ability of ALPPS to rapidly induce hyperplasia of the remaining liver is still effective even for patients who did not respond to PVE (95-98).

Patients receiving treatment to increase the remaining liver volume should be strictly limited using the following criteria: $<65$ years old, normal liver function (Child-Pugh A, ICG-R15 < 10\%), insufficient FLR (normal liver, FLR/SLV $<30 \%$; patients with chronic liver disease and liver damage, FLR/SLV $<40 \%$ ), in good general health condition, good surgical tolerance, no severe liver cirrhosis, no severe fatty liver and no severe portal hypertension (72,83,91,99-101). In addition, patients with a MELD (model for end-stage liver disease) score $>10$ are recommended to postpone the second stage of surgery (72).

Perioperative management and postoperative follow-up are very important for patients who have undergone a second surgery or surgery after conversion therapy with insufficient FLR. In addition to following the general principles of management after ordinary liver resection (102), it is necessary to focus on the characteristics of the remaining liver function in a critical state after treatment/ after the second stage excision, and accordingly pay attention to strengthening the supportive treatment of liver function to maintain the function of various organs in a good state, so as to help the remaining liver to recover steadily and further proliferate to a more fully tolerated functional volume level. At the same time, it is necessary to pay special attention to the active prevention and treatment of infections and other complications that may increase the burden of liver function.

\section{Statement 8}

In HCC patients with insufficient remaining liver volume, it is recommended that ALPPS and PVE be used in suitable populations to increase the future liver remnant; ALPPS has a higher efficacy and is preferable for use when a conversion resection is planned, but also has higher risk of perioperative complications. (Evidence-based) (GRADE: conditional recommendation, low-quality evidence. Agreement: 90.4\%).

\section{Improvement of liver function and antiviral treatment}

Abnormal liver function is a common cause of ineligibility for surgical resection. In China, the main pathogenic factors for liver cancer include hepatitis B virus (HBV) infection, hepatitis $\mathrm{C}$ virus (HCV) infection, alcoholic or nonalcoholic fatty liver disease, liver cirrhosis, and long-term exposure to carcinogens such as aflatoxin and aristolochic acid. The treatment of abnormal liver function is first of all etiological treatment, and at the same time supplemented 
with treatments such as liver protection, enzyme reduction, anti-inflammatory, repair of liver cell membrane and bile duct damage. Patients with severe liver damage or even with a liver failure tendency, often cannot tolerate antitumor therapy. These patients need to receive albumin supplementation to increase plasma colloidal osmotic pressure, and symptomatic and supportive treatments such as plasma, vitamin $\mathrm{K} 1$, and coagulation factors are required. Several studies have shown that intravenous adenosylmethionine administration 2 hours before and 57 days after hepatectomy reduced intraoperative ischemiareperfusion injury, effectively promoting postoperative liver enzyme recovery (103-105). Jaundice reduction therapy and combined adenosylmethionine drug therapy after radical surgery have been found to accelerate liver function recovery, shorten the waiting time for preoperative and other related treatments, and reduce postoperative complications (106-110). Moreover, antineoplastic therapy containing chemotherapeutic agents combined with adenosylmethionine drug therapy significantly improve patient liver function and provide hepatoprotection during subsequent chemotherapy, reducing the frequency of chemotherapy dose reductions and interruptions $(111,112)$. In addition, patients with chronic liver disease often have thrombocytopenia, which is an important indicator that affects surgical safety. Short-term treatment with thrombopoietin (TPO) receptor agonists can increase the platelet level (113), which helps to improve the resectability of liver cancer.

$\mathrm{HBV}$ infection is the leading cause of liver cancer in China, and the main cause of abnormal liver function. If $\mathrm{HBV}$-related liver cancer patients are positive for HBVDNA before surgery, whether their serum transaminase level is elevated or not, it is recommended that antiviral and hepatoprotective treatment should be given first, and surgical excision can be performed after liver function improves, so as to improve surgical safety and reduce the post-operation recurrence rate (114-116). Since immunotherapy may have a risk of inducing HBV reactivation in patients with high HBV DNA levels (i.e., $>2,000 \mathrm{kU} / \mathrm{L}$ ), it is recommended that conversion regimens including immune checkpoint inhibitors (ICIs) should be used with caution and should be included in the management of clinical trials to explore the safety of such therapies in high viral load patients. Patients with HBV DNA level $<2,000 \mathrm{kU} / \mathrm{L}$ should also receive antiviral medication throughout the course of cancer treatment, and their HBV DNA levels should be monitored. After the diagnosis of HBV-related HCC, even if HBV-DNA is negative, antiviral therapy should be initiated before receiving anti-tumor therapy, especially during anti-tumor therapy containing chemotherapeutics (such as TACE and HAIC). All HCC patients, if the relationship with viral hepatitis is unknown, should be routinely screened for $\mathrm{HBsAg}$, anti-HBc and HCV before undergoing conversion therapy for liver cancer. For confirmed HCV-associated HCC, HCV RNA should be tested. Additionally, if the HCV RNA test result is positive, DAA antiviral therapy should be administered according to the Chinese Guideline of prevention and treatment for hepatitis $C$ (2019 version). If the HCV RNA test result is negative, the HCV RNA level should be closely monitored during liver cancer conversion therapy. If HBsAg is positive, powerful, lowresistance antiviral drugs such as entecavir (ETV), tenofovir (TDF) or tenofovir alafenamide fumarate (TAF) should be initiated as soon as possible, namely one week before chemotherapy, or concurrently with chemotherapy. If $\mathrm{HBsAg}$ is negative, anti-HBc is positive, and HBV-DNA is negative, preventive antiviral therapy should be used before tumor chemotherapy, and liver function should be closely monitored. If HBV-DNA is negative, HBsAg, HBV DNA and alanine aminotransferase (ALT) levels should be monitored every 1-3 months, and if one of the first two is positive, antiviral therapy should also be conducted immediately (117).

Hepatotoxicity is one of the most common adverse reactions to ICIs and targeted combined therapy among patients with liver cancer. During treatment, if the patient has abnormal liver function, after excluding other causes, druginduced liver damage caused by ICIs needs to be considered. According to the ICIs liver damage classification standard and corresponding treatment recommended by EASL Clinical Practice Guidelines: Drug-induced liver injury (118), other comprehensive hepatoprotective protocols include: silymarin or polyene phosphatidyl choline for relatively mild inflammation; bicyclol and glycyrrhizic acid for severe inflammation; ursodeoxycholic acid for cholestatic patients. It has also been reported that adenosylmethionine has a certain effect in the treatment of cholestasis. However, the exact effect of these drugs needs to be confirmed by prospective RCTs. During the management of treatment with ICIs, cholestatic patients should also be treated with ursodeoxycholic acid and adenosylmethionine, depending on liver damage severity.

For patients with HBV-related HCC, long-term treatment with oral antiviral drugs is recommended after 
liver cancer conversion surgery. In addition, HCV-related HCC patients should be routinely monitored for HCVRNA after conversion surgery. During the entire treatment process, patient liver function should be closely monitored, and hepatoprotective drugs should be used rationally according to the degree of abnormal liver function.

\section{Statement 9}

Poor liver function is an important factor underlying unresectable HCC. Active antiviral therapy and hepatoprotective treatment should be given to improve liver function in patients with HCC who are receiving conversion therapy. Anti-tumor conversion therapy may also damage liver function. Therefore, it is necessary to closely monitor liver function and hepatitis $\mathrm{B}$ virus replication during the early treatment process, and it is recommended to conduct strong antiviral therapy throughout the course of cancer treatment. (Evidence-based) (GRADE: strong recommendation, high-quality evidence. Agreement: 96.2\%).

\section{The necessity and timing of resection}

\section{The necessity of surgical resection after successful conversion}

The significance of conversion therapy is to provide patients with opportunities for radical treatment, which in turn allows patients to achieve longer tumor-free survival and overall survival. Most of the current conversion therapy studies use short-term endpoints such as surgical resection rate and postoperative recurrence rate and there are few studies that use long-term survival as the main endpoint. A small number of retrospective studies have shown that the survival rate after conversion and resection is much higher than that associated with palliative treatments such as TACE. For example, studies conducted by Fan et al. have shown that the overall survival rates for patients with HCCat 1, 3 and 5 years after TACE conversion resection are $80 \%, 65 \%$, and $56 \%$ respectively (24). Kulik et al. found that the overall survival rates at 1,2 , and 3 years after TARE conversion resection were $84 \%, 54 \%$, and $27 \%$ respectively (119). Lewandowski et al. compared TACE and TARE as strategies for downstaging patients with HCC and reported a postoperative event-free survival of 7.1 and 17.7 months, respectively (120). Finally, a study by Zhu et al. including 63 patients with HCC found that after conversion resection $(\mathrm{n}=10)$ using TKIs combined with ICIs, after a median follow-up of 11 months, eight patients survived without tumors, and four patients had stopped medication (39). However, these retrospective studies may have selection bias, and the definition of unresectable HCC and the criteria for surgical excision are not homogeneous between the studies, which affects the comparability of survival data.

In patients with HCC receiving conversion therapy, it has been found that strong systemic treatment can lead to a pCR $(39,40,121,122)$. However, there is currently no evidence to support an association between achieving a pCR and long-term survival using continuous non-surgical treatment. Removal and then examination of all primary and metastatic lesions is the only way to ensure pCR.

For patients who have achieved a radiological response after treatment, it is uncertain whether surgery is needed. Current studies have shown that most patients achieving a response to therapy will progress within 1 to 1.5 years even with continued medication. For example, the median duration of response (DoR) of lenvatinib combined with pembrolizumab is 12.6 months, the median DoR of bevacizumab combined with atezolizumab is 18.1 months (47). In addition, from the experience of colorectal liver metastases, even if the lesions disappeared (radiological CR) after chemotherapy, more than half of the lesions will recur during the continuous follow-up process (123). Therefore, surgical excision is expected to enable patients to obtain longer tumor-free survival time and overall survival time. In addition, resection is also of great significance in reducing drug exposure and systemic treatment related adverse reactions. However, compared with chemotherapy, the depth of response of targeted therapy combined with immunotherapy may be greater. A prospective controlled study is ultimately needed to determine whether inactive lesions that disappeared on imaging or filled with contrast media after treatment still need to be surgically removed [refer to section Methods of conversion therapy-conversion therapy for tumorssystemic (drug) treatment].

\section{Statement 10}

Resection is an important means to achieve long-term survival after successful conversion therapy, but it still needs to be supported by evidence from controlled studies. Survival after conversion and resection is related to the number of surviving tumor cells in the resected tumor. Surgical resection can not only eliminate potential residual tumor cells, but also provide guidance for adjuvant 
treatment through postoperative pathological examination. (Evidence-based) (GRADE: strong recommendation, lowquality evidence. Agreement: 98.1\%).

\section{Timing of resection after successful conversion}

\section{Selection of the appropriate operation time based on tumor response}

Many scholars believe that patients with HCCwho cannot initially undergo resection due to technical reasons should undergo surgery as soon as possible once they reach the standard of surgical resectability through conversion therapy. Research shows that the tumor-free survival of patients with HCC after resection is related to the degree of pathological response, and that patients with a pathological response have longer postoperative tumor-free survival $(122,124)$. Therefore, predicting a successful conversion is based not only on the feasibility of surgical resection, but also by assessing the degree of tumor response, which is more related to the patient's postoperative recurrence and long-term survival. For technically resectable CNLC stage IIb and IIIa HCC, conversion therapy enables the tumor to achieve objective response (shrinking or downstaging) or remain stable for a period of time (such as 3-4 months) under the condition of receiving pre-conversion therapy, which may be a prerequisite for the patient to obtain better results after resection.

A major pathological response (MPR) refers to a reduction of the proportion of surviving tumors below the clinically significant value. In lung cancer and malignant melanomas, an MPR is defined as less than or equal to $10 \%$ survival tumor. Although this cut-off value is commonly used in clinical research, some studies show that the cut-off value of MPR varies in different histological types in patients receiving neoadjuvant chemotherapy. Primary liver lesions with few or no viable tumor cells with lymph node/tumor thrombus with viable metastases (ypT0, N1, 2 or 3) can also be classified as MPR, but the prognosis and therapeutic significance are still unclear (125). If the percentage of surviving tumors is close to the cut-off value of MPR after reviewing histological sections, additional histological sections should be evaluated. The pathological report should record the total number of tumor bed paraffin blocks and the proportion of residual tumor cells in each block.

A pCR refers to the complete assessment of the resected specimens, including all sampled regional lymph nodes, tumor thrombi, and distant metastases, and after reviewing all sections, no viable tumor cells were found. If there is no tumor found in the initial section and tissue of the tumor bed, additional histological slices should be made. The number of additional slices is set reasonably according to the size of the tumor bed and the ability of the pathology department. If the histological changes observed in the original slices are inconsistent with the imaging evaluation, the possibility of sampling position deviation should be considered. In this case, it may be necessary to re-evaluate the gross specimen guided by imaging. If other lesions are found, re-sampling should be carried out. The pathology report should record the total number of paraffin blocks from the tumor bed. Preliminary clinical evidence shows that patients with an MPR or pCR have better postoperative survival than those without an MPR or pCR $(39,122,124)$, however, more evidence needs to be accumulated. In addition, how to predict an MPR or pCR through imaging or other clinical indicators is also an urgent problem to be solved.

In terms of imaging evaluation, compared with the traditional RECIST v1.1 evaluation standard, the modified RECIST (mRECIST) standard was developed to better evaluate the response of liver lesions. Edeline et al. reported a group of 42 patients with HCC who were assessed as SD by RECIST v1.1. After they were re-evaluated by mRECIST, 11 patients had a CR or PR, 29 had SD and two had PD; the median OS were 17, 10 and 4 months respectively, which suggests that mRECIST can categorize tumor response in a way related to patient survival. The tumor size evaluated by the RECIST v1.1 but not just surviving tumors is of more help in judging whether a tumor is technically resectable. Although tumor response evaluation per mRECIST could be subjective to assess the extent of surviving/viable tumor, it has advantages in assessing the degree of pathological response (126).

Dynamic contrast enhanced CT and multimodal MRI have high image resolution and ability to detect HCC lesions, especially MRI has a higher detection rate for small HCC (127). Therefore, MRI is recommended as a preferred method for evaluating the efficacy of treatment. In evaluating the same lesion, for baseline and subsequent examinations, the same imaging examination method and even the same equipment should be used to reduce the system error. PET/CT or PET/MR is difficult to use as a routine imaging method for HCC due to the high examination cost. However, as a metabolic function imaging method, it can still play a useful role in evaluating the therapeutic effect of non-cytotoxic drugs.

\section{Statement 11}

The efficacy of conversion and resection of HCC is related 
to the number of surviving tumor cells in the tumor tissue. Therefore, an important condition for conversion and resection is to obtain a tumor response, or at least to keep the lesion stable for a period of time (3 to 4 months). Tumor response evaluated by mRECIST may be more related to pathological changes. The use of imaging detection methods to predict the pathological response of tumors is a subject that needs further research. (Based on expert opinion) (GRADE: strong recommendation, low-quality evidence. Agreement: 96.2\%).

\section{Choosing the appropriate operation time based on safety considerations}

The safety of surgical resection should be considered when deciding on the timing of resection. Different preoperative treatment methods require different timing of resection. For system treatment, few studies have clarified the optimal time to stop drug therapy before surgery. There is no clear preoperative withdrawal time for patients who are receiving small-molecule targeted drugs. According to the existing literature, continuous medication before surgery will not lead to an increase of postoperative complications $(128,129)$. In some case reports, surgery was performed after stopping the drug for 1 week before surgery (130). The half-life of bevacizumab is about 20 days, and its antiangiogenic effect may lead to an increased risk of bleeding and reduced wound healing capacity (131). From the experience of colorectal liver metastases liver resection, it is generally necessary to stop bevacizumab for 46 weeks before surgery to ensure the safety of hepatectomy. Immunotherapy is given every 2-3 weeks. Some literature reports suggest that HCC conversion surgery should be performed within 4 weeks after the end of the last drug cycle $(132,133)$. If adverse events occur during targeted or immunotherapy, surgery should be performed when adverse events return to grade I or normal. At present, there is no data on the effect of anti-angiogenic drugs combined with PD-1 inhibitors on postoperative liver function and surgical safety. Furthermore, the safety assessment of surgery in patients treated with PD-1 monoclonal antibodies must take into consideration the presence/absence of immune hepatitis, which is likely to lead to an increased risk of surgical death. For immune hepatitis evaluation, in addition to the conventional hepatocellular injury markers such as ALT and AST, liver aspiration biopsy can be performed to observe inflammatory and lymphocyte infiltration and hepatocyte necrosis. The results of studies on the effect of anti-angiogenic drugs combined with PD-1 inhibitors on postoperative liver function and the safety of surgery are scarce and more data are required to determine a reasonable preoperative assessment strategy.

TACE is the main treatment for advanced HCC and is considered to be the standard treatment for bridging therapy before liver transplantation. Its role in conversion therapy and neoadjuvant therapy of HCC is also fully recognized. Previous studies have found that preoperative TACE can cause liver inflammation, increase intraoperative blood loss and lead to operation difficulty (134). However, it has been noted that when the interval between the last round of TACE and surgery is long enough, TACE has minimal impact on the operation. It is recommended that the preoperative interval between the last round of TACE and surgery should be at least 4 weeks (median time interval is 6 weeks), and this will have no significant effect on the perioperative complication rate, mortality rate, etc. $(135,136)$. Although some patients have perihepatitis adhesions within a few weeks after TACE, these have no obvious negative impact on surgery or the short-term prognosis of the operation. In some patients receiving TACE combined with PVE as conversion therapy, data recommends that the remaining liver volume be evaluated every 2 weeks after the end of PVE, and surgery can be performed when the remaining liver volume and ICG-R15 reach the indications for surgery (137). The liver function is likely to be damaged during interventional treatment, and surgery should be performed after the liver function is stable.

Radiotherapy is an important neoadjuvant therapy for HCC, especially in the control of portal vein and hepatic vein tumor thrombus. In a study of preoperative radiotherapy for surgically resectable HCC with portal vein tumor thrombus in China, surgery within 5 weeks after the end of preoperative stereotactic radiotherapy (SBRT) resulted in good surgical outcomes and improved the longterm prognosis of patients. The best time interval between main portal vein radiotherapy and hemihepatectomy is 4 weeks, which can minimize the incidence of liver function damage, intraoperative blood loss and postoperative liver failure (138).

\section{Statement 12}

The safety of surgery is an important consideration to be evaluated before conversion resection. These assessments not only require evaluation of the safety examination items necessary for general hepatectomy, but also require assessment of the potential impact of early conversion 
therapy on the liver. Before surgery, small molecule targeted drugs (such as lenvatinib, apatinib, sorafenib, etc.) should be suspended for more than 1-2 weeks, PD-1 inhibitors should be suspended for more than 2-4 weeks and bevacizumab should be suspended for more than 6 weeks. If TACE or radiotherapy is performed, the operation should be performed 4 weeks after the last treatment. (Evidence-based) (GRADE: strong recommendation, low-quality evidence. Agreement: 94.2\%).

\section{Multi-disciplinary teams are important to ensure the quality of conversion therapy}

The methods currently used for conversion therapy of HCC include local therapy (vascular intervention, radiotherapy, etc.) and systemic drugs. Different treatment methods have their own advantages and disadvantages, and the indications overlap. The implementation of treatment methods involves multiple disciplines. At the same time, the biological behavior of HCC is highly heterogeneous, and the liver disease backgrounds and prognostic factors of different individuals are quite different. Moreover, due to the short implementation time of conversion therapy of HCC, there is still a lack of clear guidelines and norms on its applicable population, specific methods and means, the timing of surgery after conversion therapy, and management of adverse reactions during the process of treatment. It is necessary to gradually summarize experience in practice and the different departments involved in treatment should repeatedly communicate and discuss and form a consensus while reserving differences. Therefore, the cooperation and communication among the multidisciplinary team (MDT) in the process of conversion therapy of HCC is very important. A relatively fixed MDT team must be established to form a smooth and convenient communication channel to ensure that the treatment plan can be adjusted in a timely manner according to the patient's condition, so as to maximize the benefit of patients.

The short-term goal of conversion therapy of liver cancer is to create opportunities for radical surgery, and the ultimate goal is to enable patients to obtain high-quality long-term survival. In the process of translation therapy, medical personnel of all disciplines should keep in mind that resection is not the only purpose of conversion, and follow the principle of "three musts and three don'ts" of a HCC MDT (must be patient-centered, must be curativeeffect-oriented, must be evidence-based; don't use your own experience to determine the patient's treatment plan, don't use too many single treatments, don't decide the treatment plan based on economic benefits), and provide individualized optimal decisions for HCC patients through an MDT.

\section{Statement 13}

Although resection is the main goal of conversion therapy, it is not the final goal in treating HCC patients. Multidisciplinary discussion is necessary for implementing conversion therapy and deciding the treatment strategy after conversion therapy. Maximizing patient benefit should be the only goal of MDT cooperation. (Based on expert opinion) (GRADE: strong recommendation, low-quality evidence. Agreement: 100\%).

\section{Other issues to be explored}

\section{Adjuvant treatment after conversion surgery}

There remains a lack of high-level evidence for the selection of post-operative treatment after conversion and R0 resection. However, the success of conversion therapy not only gives the initial unresectable HCC a chance of radical resection, but also confirms that the tumor is sensitive to conversion therapy, and it is reasonable to use the pre-operative regime for post-operative treatment. The choice of postoperative treatment regimen should be deliberated in light of effectiveness and safety. If the conversion treatment plan is a combination of drugs and local treatments (radiotherapy, HAIC, etc.), since the target lesions were surgically removed, the systemic treatment may be the choice of post-operative treatment. If the conversion treatment plan is a combination of multiple drugs, such as targeted and immunotherapy, dual immune therapy combination, etc., the original plan or certain drugs in the original plan should be selected according to the patient's physical performance and adverse reactions associated with the systemic treatment.

There is also no sufficient data on the optimal duration of adjuvant therapy. Based on previous studies on adjuvant therapy and the progression-free survival of systemic therapy, it is recommended that postoperative adjuvant therapy should last for more than 6 months with a close observation every 2-3 months. If there are no signs of tumor recurrence and metastasis in two consecutive imaging examinations and the tumor markers (AFP, PIVKA-II) are not increased, the adjuvant therapy can be 
suspended. Adverse reactions should be closely monitored during adjuvant treatment. Once serious adverse reactions occur or the patient cannot tolerate treatment, the medication dose should be reduced or suspended. For patients whose resected tumor specimens show a pCR, a shorter postoperative adjuvant treatment duration can be considered.

\section{Statement 14}

After R0 resection, the conversion therapy regimen should be used as adjuvant treatment for at least 6 months according to patient's performance status, adverse reactions and treatment tolerance of the patient. Drug withdrawal may be considered if there is no recurrence or metastasis in two consecutive imaging examinations and tumor markers remain normal. (Based on expert opinion) (GRADE: conditional recommendation, low-quality evidence. Agreement: $86.5 \%)$.

\section{Treatment for patients not achieving conversion}

Non-responding or progressive tumors, insufficient growth of future liver remnant and deterioration of underlying liver disease usually result in failure of conversion therapy. For patients experiencing deterioration of basic liver disease (including serious adverse events related to systematic treatment), the treatment options are active supportive and symptomatic treatment. The main reason for the failure of conversion therapy is that systemic therapy or local therapy fails to achieve a tumor response, next line treatment needs to be explored according to the initial treatment and the pattern of disease progression.

Some patients may have rapid disease progression on first-line systemic treatment. Systemic chemotherapy can be considered for patients who have used ICIs combined with TKIs; patients who have used ICIs combined with bevacizumab can be treated with other anti-proliferative drugs [such as TKIs (139) or systemic chemotherapy]. For patients with slow progression, it is recommended to treat them according to the patterns of progression: (I) if extrahepatic lesions are stable and only intrahepatic primary target lesions progress, local treatment for intrahepatic lesions, such as TACE or HAIC, is recommended; if new lesions appear in the liver, but other lesions are stable, local treatment, such as radiofrequency ablation, can be applied to the new lesions; (II) if both intrahepatic and extrahepatic lesions progressed, it is suggested to adjust to second-line systemic therapy, and combination or single drug regimens with different mechanisms of action can be selected sequentially; (III) radiotherapy for portal vein tumor thrombus is recommended if the new tumor thrombus or the original tumor thrombus develops while other target lesions are stable. It is worth noting that the evolution process of advanced HCC is complex, so close follow-up is needed. If necessary, the assessment interval should be shortened (4-6 weeks), and the changes of patient symptoms and corresponding tumor markers should be taken into account.

\section{Statement 15}

When selecting subsequent therapy for patients who fail conversion therapy, it is necessary to take into account the basic liver disease, initial treatment methods, patterns of tumor progression and patients' preference. Second-line treatment should be selected according to the patterns of disease progression. (Based on expert opinion) (GRADE: conditional recommendation, low-quality evidence. Agreement: 96.2\%).

\section{Application of conversion therapy in patients with HCC complicated with extrahepatic metastasis}

HCC with extrahepatic metastasis refers to lymph node metastasis or distant metastasis, including lung, bone, brain, diaphragm, adrenal gland and omentum metastasis. Lung is the most common organ for extrahepatic metastasis, accounting for $67.3 \%$ of all extrahepatic metastasis, followed by abdominal lymph nodes $(37.5 \%)$, bone (18.3\%) and adrenal gland (7.6\%) (140). Systemic therapy is the first choice for patients with extrahepatic metastasis. A number of retrospective studies have shown that most (70-80\%) patients with HCC complicated with extrahepatic metastasis die of liver failure caused by intrahepatic tumor progression, rather than extrahepatic metastasis (140-143). According to the indications for resection recommended in the Chinese Guidelines for the Diagnosis and Treatment of Hepatocellular Carcinoma (2019 Edition), patients with extrahepatic metastasis can also accept surgical resection under specific circumstances (7). In recent years, a number of studies have shown that resection of lung metastases may improve the survival of HCC patients with lung metastases, especially for patients after intrahepatic primary tumor resection, liver transplantation or patients whose primary tumor is well controlled and extrahepatic metastases only 
involve the lung and include less than 3 metastatic nodules. The median tumor-free survival time after resection of lung metastases is 7-38 months, and the median overall survival is 16-52 months (144-150). In addition, there have been several reports showing resection of HCC with local lymph node metastasis and adrenal metastasis may have survival benefit. These studies show that the median survival time after surgical resection of lymph node and adrenal metastasis is 29 and 21 months, respectively $(151,152)$. However, the current research on resection of extrahepatic lesions for patients with HCC and distant metastasis includes small patient samples, and there is a lack of evidence for conversion therapy specifically for patients with extrahepatic metastasis. Therefore, the application of conversion therapy for patients with HCC and extrahepatic metastasis requires further research.

\section{Statement 16}

Systemic therapy is the first choice for patients with extrahepatic metastasis. Some patients with extrahepatic metastases may gain a survival benefit through primary liver resection after conversion therapy and control of extrahepatic metastases, but further research is required. (Based on expert opinion) (GRADE: strong recommendation, low-quality evidence. Agreement: 92.3\%).

\section{Acknowledgments}

The authors would like to thank the China Liver Cancer Society of China Anti-Cancer Society for supporting this work.

Funding: None.

\section{Footnote}

Reporting Checklist: The authors have completed the RIGHT reporting checklist. Available at https://hbsn. amegroups.com/article/view/10.21037/hbsn-21-328/rc

Conflicts of Interest: All authors have completed the ICMJE uniform disclosure form (available at https://hbsn. amegroups.com/article/view/10.21037/hbsn-21-328/coif). YM serves as the Editor-in-Chief and HZ serves as an unpaid Deputy Editor-in-Chief of Hepatobiliary Surgery and Nutrition. The other authors have no conflicts of interest to declare.
Etbical Statement: The authors are accountable for all aspects of the work in ensuring that questions related to the accuracy or integrity of any part of the work are appropriately investigated and resolved.

Open Access Statement: This is an Open Access article distributed in accordance with the Creative Commons Attribution-NonCommercial-NoDerivs 4.0 International License (CC BY-NC-ND 4.0), which permits the noncommercial replication and distribution of the article with the strict proviso that no changes or edits are made and the original work is properly cited (including links to both the formal publication through the relevant DOI and the license). See: https://creativecommons.org/licenses/by-nc-nd/4.0/.

\section{References}

1. Jaeschke R, Guyatt GH, Dellinger P, et al. Use of GRADE grid to reach decisions on clinical practice guidelines when consensus is elusive. BMJ 2008;337:a744.

2. Aguayo-Albasini JL, Flores-Pastor B, Soria-Aledo V. GRADE system: classification of quality of evidence and strength of recommendation. Cir Esp 2014;92:82-8.

3. Bray F, Ferlay J, Soerjomataram I, et al. Global cancer statistics 2018: GLOBOCAN estimates of incidence and mortality worldwide for 36 cancers in 185 countries. CA Cancer J Clin 2018;68:394-424.

4. Forner A, Reig M, Bruix J. Hepatocellular carcinoma. Lancet 2018;391:1301-14.

5. Yang JD, Hainaut P, Gores GJ, et al. A global view of hepatocellular carcinoma: trends, risk, prevention and management. Nat Rev Gastroenterol Hepatol 2019;16:589-604.

6. Chen W, Zheng R, Baade PD, et al. Cancer statistics in China, 2015. CA Cancer J Clin 2016;66:115-32.

7. Zhou J, Sun H, Wang Z, et al. Guidelines for the Diagnosis and Treatment of Hepatocellular Carcinoma (2019 Edition). Liver Cancer 2020;9:682-720.

8. Foerster F, Galle PR. Comparison of the current international guidelines on the management of HCC. JHEP Rep 2019;1:114-9.

9. Park JW, Chen M, Colombo M, et al. Global patterns of hepatocellular carcinoma management from diagnosis to death: the BRIDGE Study. Liver Int 2015;35:2155-66.

10. Kudo M, Finn RS, Qin S, et al. Lenvatinib versus sorafenib in first-line treatment of patients with unresectable hepatocellular carcinoma: a randomised phase 3 non- 
inferiority trial. Lancet 2018;391:1163-73.

11. Kloeckner R, Galle PR, Bruix J. Local and Regional Therapies for Hepatocellular Carcinoma. Hepatology 2021;73 Suppl 1:137-49.

12. Kudo M, Cheng AL, Park JW, et al. Orantinib versus placebo combined with transcatheter arterial chemoembolisation in patients with unresectable hepatocellular carcinoma (ORIENTAL): a randomised, double-blind, placebo-controlled, multicentre, phase 3 study. Lancet Gastroenterol Hepatol 2018;3:37-46.

13. Zhong JH, Ke Y, Gong WF, et al. Hepatic resection associated with good survival for selected patients with intermediate and advanced-stage hepatocellular carcinoma. Ann Surg 2014;260:329-40.

14. Wang K, Guo WX, Chen MS, et al. Multimodality Treatment for Hepatocellular Carcinoma With Portal Vein Tumor Thrombus: A Large-Scale, Multicenter, Propensity Mathching Score Analysis. Medicine (Baltimore) 2016;95:e3015.

15. Xia YX, Zhang F, Li X, et al. Surgical treatment of primary liver cancer: a report of 10966 cases. Chin J Surg 2021;59:6-17.

16. Finn RS, Ikeda M, Zhu AX, et al. Phase Ib Study of Lenvatinib Plus Pembrolizumab in Patients With Unresectable Hepatocellular Carcinoma. J Clin Oncol 2020;38:2960-70.

17. Finn RS, Qin S, Ikeda M, et al. Atezolizumab plus Bevacizumab in Unresectable Hepatocellular Carcinoma. N Engl J Med 2020;382:1894-905.

18. Xu J, Shen J, Gu S, et al. Camrelizumab in Combination with Apatinib in Patients with Advanced Hepatocellular Carcinoma (RESCUE): A Nonrandomized, Open-label, Phase II Trial. Clin Cancer Res 2021;27:1003-11.

19. Yau T, Kang YK, Kim TY, et al. Efficacy and Safety of Nivolumab Plus Ipilimumab in Patients With Advanced Hepatocellular Carcinoma Previously Treated With Sorafenib: The CheckMate 040 Randomized Clinical Trial. JAMA Oncol 2020;6:e204564.

20. He M, Li Q, Zou R, et al. Sorafenib Plus Hepatic Arterial Infusion of Oxaliplatin, Fluorouracil, and Leucovorin vs Sorafenib Alone for Hepatocellular Carcinoma With Portal Vein Invasion: A Randomized Clinical Trial. JAMA Oncol 2019;5:953-60.

21. He MK, Le Y, Li QJ, et al. Hepatic artery infusion chemotherapy using mFOLFOX versus transarterial chemoembolization for massive unresectable hepatocellular carcinoma: a prospective non-randomized study. Chin J Cancer 2017;36:83.
22. Pan YX, Fu YZ, Hu DD, et al. Stereotactic Body Radiotherapy vs. Radiofrequency Ablation in the Treatment of Hepatocellular Carcinoma: A Meta-Analysis. Front Oncol 2020;10:1639.

23. Hermann RE, Lonsdale D. Chemotherapy, radiotherapy, and hepatic lobectomy for hepatoblastoma in an infant: report of a survival. Surgery 1970;68:383-8.

24. Fan J, Tang ZY, Yu YQ, et al. Improved survival with resection after transcatheter arterial chemoembolization (TACE) for unresectable hepatocellular carcinoma. Dig Surg 1998;15:674-8.

25. Lau WY, Ho SK, Yu SC, et al. Salvage surgery following downstaging of unresectable hepatocellular carcinoma. Ann Surg 2004;240:299-305.

26. Sitzmann JV, Abrams R. Improved survival for hepatocellular cancer with combination surgery and multimodality treatment. Ann Surg 1993;217:149-54.

27. Tang ZY, Liu KD, Bao YM, et al. Radioimmunotherapy in the multimodality treatment of hepatocellular carcinoma with reference to second-look resection. Cancer 1990;65:211-5.

28. Zhang Y, Huang G, Wang Y, et al. Is Salvage Liver Resection Necessary for Initially Unresectable Hepatocellular Carcinoma Patients Downstaged by Transarterial Chemoembolization? Ten Years of Experience. Oncologist 2016;21:1442-9.

29. Tang ZY, Uy YQ, Zhou XD, et al. Cytoreduction and sequential resection for surgically verified unresectable hepatocellular carcinoma: evaluation with analysis of 72 patients. World J Surg 1995;19:784-9.

30. Zhao HT, Sang XT, Rui JA, et al. Analysis of the curative effect of resection for unresectable advanced liver cancer after down-stage. Acta Academiae Medicinae Sinicae 2009;31:503-5.

31. Torzilli G, Belghiti J, Kokudo N, et al. A snapshot of the effective indications and results of surgery for hepatocellular carcinoma in tertiary referral centers: is it adherent to the EASL/AASLD recommendations?: an observational study of the HCC East-West study group. Ann Surg 2013;257:929-37.

32. Yin L, Li H, Li AJ, et al. Partial hepatectomy vs. transcatheter arterial chemoembolization for resectable multiple hepatocellular carcinoma beyond Milan Criteria: a RCT. J Hepatol 2014;61:82-8.

33. Wang Q, Xia D, Bai W, et al. Development of a prognostic score for recommended TACE candidates with hepatocellular carcinoma: A multicentre observational study. J Hepatol 2019;70:893-903. 
34. Bolondi L, Burroughs A, Dufour JF, et al. Heterogeneity of patients with intermediate (BCLC B) Hepatocellular Carcinoma: proposal for a subclassification to facilitate treatment decisions. Semin Liver Dis 2012;32:348-59.

35. Lei Y, Yee LW, Zhou WP. Multiple hepatocellular carcinomas: liver resection or transcatheter arterial chemoembolization? Hepatobiliary Surg Nutr 2019;8:519-21.

36. Kudo M, Ueshima K, Chan S, et al. Lenvatinib as an Initial Treatment in Patients with Intermediate-Stage Hepatocellular Carcinoma Beyond Up-To-Seven Criteria and Child-Pugh A Liver Function: A Proof-Of-Concept Study. Cancers (Basel) 2019;11:1084.

37. Chen $\mathrm{X}$, Zhang $\mathrm{Y}$, Zhang $\mathrm{N}$, et al. Lenvatinib combined nivolumab injection followed by extended right hepatectomy is a feasible treatment for patients with massive hepatocellular carcinoma: a case report. Onco Targets Ther 2019;12:7355-9.

38. Kolligs FT, Bilbao JI, Jakobs T, et al. Pilot randomized trial of selective internal radiation therapy vs. chemoembolization in unresectable hepatocellular carcinoma. Liver Int 2015;35:1715-21.

39. Zhu XD, Huang C, Shen YH, et al. Downstaging and Resection of Initially Unresectable Hepatocellular Carcinoma with Tyrosine Kinase Inhibitor and Anti-PD-1 Antibody Combinations. Liver Cancer 2021;10:320-9.

40. Zhang WW, Hu BY, Han J, et al. Preliminary report on the study of conversion therapy of advanced hepatocellular carcinoma combined PD-1 inhibitors with multitarget tyrosine kinase inhibitors. Chinese Journal of Hepatobiliary Surgery 2020;26:947-8.

41. Ren Z, Xu J, Bai Y, et al. Sintilimab plus a bevacizumab biosimilar (IBI305) versus sorafenib in unresectable hepatocellular carcinoma (ORIENT-32): a randomised, open-label, phase 2-3 study. Lancet Oncol 2021;22:97790. Erratum in: Lancet Oncol 2021;22:e347.

42. Kudo M, Ikeda M, Motomura K, et al. A phase Ib study of lenvatinib (LEN) plus nivolumab (NIV) in patients (pts) with unresectable hepatocellular carcinoma (uHCC): Study 117. J Clin Oncol 2020;38:abstr 513.

43. Galle PR, Kim RD, Sung MW, et al. 990P Updated results of a phase Ib study of regorafenib (REG) plus pembrolizumab (PEMBRO) for first-line treatment of advanced hepatocellular carcinoma (HCC). Ann Oncol 2020;31:S691-S692.

44. Yau T, Zagonel V, Santoro A, et al. Nivolumab (NIVO) + ipilimumab (IPI) + cabozantinib (CABO) combination therapy in patients (pts) with advanced hepatocellular carcinoma (aHCC): Results from CheckMate 040. J Clin Oncol 2020;38:abstr 478.

45. Jiao SC, Bai L, Dong J, et al. Clinical activity and safety of penpulimab (Anti-PD-1) with anlotinib as first-line therapy for advanced hepatocellular carcinoma (HCC). J Clin Oncol 2020;38:abstr 4592.

46. Hao C, Du C, Peng B, et al. Toripalimab plus bevacizumab as first-line treatment for advanced hepatocellular carcinoma: A single-arm phase II study. ASCO GI 2022. Abstract 435\#.

47. Finn RS, Qin S, Ikeda M, et al. IMbrave150: Updated overall survival (OS) data from a global, randomized, open-label phase III study of atezolizumab (atezo) + bevacizumab (bev) versus sorafenib (sor) in patients (pts) with unresectable hepatocellular carcinoma (HCC). J Clin Oncol 2021;39:abstr 267.

48. He AR, Yau T, Hsu C, et al. Nivolumab (NIVO) + ipilimumab (IPI) combination therapy in patients (pts) with advanced hepatocellular carcinoma (aHCC): Subgroup analyses from CheckMate 040. J Clin Oncol 2020;38:abstr 512.

49. Qin S, Chen Z, Liu Y, et al. A phase II study of anti-PD-1 antibody camrelizumab plus FOLFOX4 or GEMOX systemic chemotherapy as first-line therapy for advanced hepatocellular carcinoma or biliary tract cancer. J Clin Oncol 2019;37:abstr 4074.

50. Song X, Kelley RK, Khan A, et al. Exposure-response (ER) efficacy and safety (E-S) analyses of tremelimumab as monotherapy or in combination with durvalumab in patients (pts) with unresectable hepatocellular carcinoma (uHCC). J Clin Oncol 2021;39:abstr 313.

51. Shi M, Li Q, He M, et al. $981 O$ Hepatic arterial infusion chemotherapy (HAIC) with oxaliplatin, fluorouracil, and leucovorin (FOLFOX) versus transarterial chemoembolization (TACE) for unresectable hepatocellular carcinoma (HCC): A randomised phase III trial. Ann Oncol 2020;31:S688.

52. Meyer T, Fox R, Ma YT, et al. Sorafenib in combination with transarterial chemoembolisation in patients with unresectable hepatocellular carcinoma (TACE 2): a randomised placebo-controlled, double-blind, phase 3 trial. Lancet Gastroenterol Hepatol 2017;2:565-75.

53. Stein S, Hsu CH, Lee M, et al. Patterns of response to atezolizumab (atezo) + bevacizumab (bev) in hepatocellular carcinoma (HCC) from the Phase 1b GO30140 study. EASL Digital Liver Cancer Summit 2021;P003.

54. Huang C, Zhu XD, Shen YH, et al. Organ specific responses to first-line lenvatinib plus anti-PD-1 antibodies 
in patients with unresectable hepatocellular carcinoma: a retrospective analysis. Biomark Res 2021;9:19.

55. Kim Y, Stahl CC, Makramalla A, et al. Downstaging therapy followed by liver transplantation for hepatocellular carcinoma beyond Milan criteria. Surgery 2017;162:1250-8.

56. Llovet JM, Real MI, Montaña X, et al. Arterial embolisation or chemoembolisation versus symptomatic treatment in patients with unresectable hepatocellular carcinoma: a randomised controlled trial. Lancet 2002;359:1734-9.

57. Lo CM, Ngan H, Tso WK, et al. Randomized controlled trial of transarterial lipiodol chemoembolization for unresectable hepatocellular carcinoma. Hepatology 2002;35:1164-71.

58. Orlacchio A, Chegai F, Merolla S, et al. Downstaging disease in patients with hepatocellular carcinoma outside up-to-seven criteria: Strategies using degradable starch microspheres transcatheter arterial chemo-embolization. World J Hepatol 2015;7:1694-700.

59. Li B, Qiu J, Zheng Y, et al. Conversion to Resectability Using Transarterial Chemoembolization Combined With Hepatic Arterial Infusion Chemotherapy for Initially Unresectable Hepatocellular Carcinoma. Annals of Surgery Open 2021;2:e057.

60. Marcacuzco Quinto A, Nutu OA, San Román Manso R, et al. Complications of transarterial chemoembolization (TACE) in the treatment of liver tumors. Cir Esp (Engl Ed) 2018;96:560-7.

61. Choi JH, Chung WJ, Bae SH, et al. Randomized, prospective, comparative study on the effects and safety of sorafenib vs. hepatic arterial infusion chemotherapy in patients with advanced hepatocellular carcinoma with portal vein tumor thrombosis. Cancer Chemother Pharmacol 2018;82:469-78.

62. Lyu N, Kong Y, Mu L, et al. Hepatic arterial infusion of oxaliplatin plus fluorouracil/leucovorin vs. sorafenib for advanced hepatocellular carcinoma. J Hepatol 2018;69:60-9.

63. He MK, Liang RB, Zhao Y, et al. Lenvatinib, toripalimab, plus hepatic arterial infusion chemotherapy versus lenvatinib alone for advanced hepatocellular carcinoma. Ther Adv Med Oncol 2021;13:17588359211002720.

64. Lencioni R, de Baere T, Soulen MC, et al. Lipiodol transarterial chemoembolization for hepatocellular carcinoma: A systematic review of efficacy and safety data. Hepatology 2016;64:106-16.

65. Lau WY, Ho S, Leung TW, et al. Selective internal radiation therapy for nonresectable hepatocellular carcinoma with intraarterial infusion of 90yttrium microspheres. Int J Radiat Oncol Biol Phys 1998;40:583-92.

66. Salem R, Lewandowski RJ, Mulcahy MF, et al. Radioembolization for hepatocellular carcinoma using Yttrium-90 microspheres: a comprehensive report of longterm outcomes. Gastroenterology 2010;138:52-64.

67. Kokabi N, Camacho JC, Xing M, et al. Open-label prospective study of the safety and efficacy of glass-based yttrium 90 radioembolization for infiltrative hepatocellular carcinoma with portal vein thrombosis. Cancer 2015;121:2164-74.

68. Kamiyama T, Nakanishi K, Yokoo H, et al. Efficacy of preoperative radiotherapy to portal vein tumor thrombus in the main trunk or first branch in patients with hepatocellular carcinoma. Int J Clin Oncol 2007;12:363-8.

69. Wei X, Jiang Y, Zhang X, et al. Neoadjuvant ThreeDimensional Conformal Radiotherapy for Resectable Hepatocellular Carcinoma With Portal Vein Tumor Thrombus: A Randomized, Open-Label, Multicenter Controlled Study. J Clin Oncol 2019;37:2141-51.

70. Lee HS, Choi GH, Choi JS, et al. Surgical resection after down-staging of locally advanced hepatocellular carcinoma by localized concurrent chemoradiotherapy. Ann Surg Oncol 2014;21:3646-53.

71. Byun HK, Kim HJ, Im YR, et al. Dose escalation by intensity modulated radiotherapy in liver-directed concurrent chemoradiotherapy for locally advanced BCLC stage $C$ hepatocellular carcinoma. Radiother Oncol 2019;133:1-8.

72. Oldhafer KJ, Stavrou GA, van Gulik TM, et al. ALPPS-Where Do We Stand, Where Do We Go?: Eight Recommendations From the First International Expert Meeting. Ann Surg 2016;263:839-41.

73. Suda K, Ohtsuka M, Ambiru S, et al. Risk factors of liver dysfunction after extended hepatic resection in biliary tract malignancies. Am J Surg 2009;197:752-8.

74. Vauthey JN, Dixon E, Abdalla EK, et al. Pretreatment assessment of hepatocellular carcinoma: expert consensus statement. HPB (Oxford) 2010;12:289-99.

75. Professional Committee of Hepatobiliary and Pancreatic Surgery of Chinese Research Hospital Association. Expert consensus on precision liver resection. Chinese Journal of Gastroenterology Surgery 2017;16:883-93.

76. Bureau of Medical Administration, National Health and Family Planning Commission of the PRC. Standardization of diagnosis and treatment for hepatocellular carcinoma 
(2017 edition). Chin J Dig Surg 2017,16:635-47.

77. Imamura H, Sano K, Sugawara Y, et al. Assessment of hepatic reserve for indication of hepatic resection: decision tree incorporating indocyanine green test. J Hepatobiliary Pancreat Surg 2005;12:16-22.

78. Shindoh J, D Tzeng CW, Vauthey JN. Portal vein embolization for hepatocellular carcinoma. Liver Cancer 2012;1:159-67.

79. Aloia TA. Associating Liver Partition and Portal Vein Ligation for Staged Hepatectomy: Portal Vein Embolization Should Remain the Gold Standard. JAMA Surg 2015;150:927-8.

80. Piron L, Deshayes E, Escal L, et al. Portal vein embolization: Present and future. Bull Cancer 2017;104:407-16.

81. European Association for Study of Liver; European Organisation for Research and Treatment of Cancer. EASL-EORTC clinical practice guidelines: management of hepatocellular carcinoma. Eur J Cancer 2012;48:599-641.

82. Shindoh J, Vauthey JN, Zimmitti G, et al. Analysis of the efficacy of portal vein embolization for patients with extensive liver malignancy and very low future liver remnant volume, including a comparison with the associating liver partition with portal vein ligation for staged hepatectomy approach. J Am Coll Surg 2013;217:126-33; discussion 133-4.

83. Sun $Z$, Tang $W$, Sakamoto $Y$, et al. A systematic review and meta-analysis of feasibility, safety and efficacy of associating liver partition and portal vein ligation for staged hepatectomy (ALPPS) versus two-stage hepatectomy (TSH). Biosci Trends 2015;9:284-8.

84. Ogata S, Belghiti J, Farges O, et al. Sequential arterial and portal vein embolizations before right hepatectomy in patients with cirrhosis and hepatocellular carcinoma. $\mathrm{Br} \mathrm{J}$ Surg 2006;93:1091-8.

85. Hwang S, Ha TY, Ko GY, et al. Preoperative Sequential Portal and Hepatic Vein Embolization in Patients with Hepatobiliary Malignancy. World J Surg 2015;39:2990-8.

86. Dupré A, Hitier M, Peyrat P, et al. Associating portal embolization and artery ligation to induce rapid liver regeneration in staged hepatectomy. Br J Surg 2015;102:1541-50.

87. Glantzounis GK, Tokidis E, Basourakos SP, et al. The role of portal vein embolization in the surgical management of primary hepatobiliary cancers. A systematic review. Eur J Surg Oncol 2017;43:32-41.

88. Chinese Anti-Cancer Association. Expert consensus on standardized diagnosis and treatment of hilar cholangiocarcinoma (2015). Chinese Journal of Hepatobiliary Surgery 2015;21:505-11.

89. Lau WY, Lai EC. Salvage surgery following downstaging of unresectable hepatocellular carcinoma--a strategy to increase resectability. Ann Surg Oncol 2007;14:3301-9.

90. Popescu GA, Alexandrescu ST, Grigorie RT, et al. GOOD TO KNOW: The ALPPS Procedure - Embracing a New Technique. Chirurgia (Bucur) 2017;112:332-41.

91. Schadde E, Ardiles V, Robles-Campos R, et al. Early survival and safety of ALPPS: first report of the International ALPPS Registry. Ann Surg 2014;260:829-36; discussion 836-8.

92. Schnitzbauer AA, Lang SA, Goessmann H, et al. Right portal vein ligation combined with in situ splitting induces rapid left lateral liver lobe hypertrophy enabling 2 -staged extended right hepatic resection in small-for-size settings. Ann Surg 2012;255:405-14.

93. Wang Z, Peng Y, Hu J, et al. Associating Liver Partition and Portal Vein Ligation for Staged Hepatectomy for Unresectable Hepatitis B Virus-related Hepatocellular Carcinoma: A Single Center Study of 45 Patients. Ann Surg 2020;271:534-41.

94. Li PP, Huang G, Jia NY, et al. Associating liver partition and portal vein ligation for staged hepatectomy versus sequential transarterial chemoembolization and portal vein embolization in staged hepatectomy for HBV-related hepatocellular carcinoma: a randomized comparative study. HepatoBiliary Surg Nutr 2020. doi: 10.21037/ hbsn-20-264

95. Enne M, Schadde E, Björnsson B, et al. ALPPS as a salvage procedure after insufficient future liver remnant hypertrophy following portal vein occlusion. HPB (Oxford) 2017;19:1126-9.

96. Sandström P, Røsok BI, Sparrelid E, et al. ALPPS Improves Resectability Compared With Conventional Two-stage Hepatectomy in Patients With Advanced Colorectal Liver Metastasis: Results From a Scandinavian Multicenter Randomized Controlled Trial (LIGRO Trial). Ann Surg 2018;267:833-40.

97. Tschuor Ch, Croome KP, Sergeant G, et al. Salvage parenchymal liver transection for patients with insufficient volume increase after portal vein occlusion -an extension of the ALPPS approach. Eur J Surg Oncol 2013;39:1230-5.

98. Ulmer TF, de Jong C, Andert A, et al. ALPPS Procedure in Insufficient Hypertrophy After Portal Vein Embolization (PVE). World J Surg 2017;41:250-7.

99. Buac S, Schadde E, Schnitzbauer AA, et al. The many 
faces of ALPPS: surgical indications and techniques among surgeons collaborating in the international registry. HPB (Oxford) 2016;18:442-8.

100.D'Haese JG, Neumann J, Weniger M, et al. Should ALPPS be Used for Liver Resection in Intermediate-Stage HCC? Ann Surg Oncol 2016;23:1335-43.

101. Vennarecci G, Laurenzi A, Levi Sandri GB, et al. The ALPPS procedure for hepatocellular carcinoma. Eur J Surg Oncol 2014;40:982-8.

102. Chen XP, Mao YL, Qiu YD, et al. Expert consensus on perioperative management of hepatectomy. Chinese Journal of Practical Surgery 2017;05:69-74.

103. Liu GY, Wang W, Jia WD, et al. Protective effect of $\mathrm{S}$-adenosylmethionine on hepatic ischemia-reperfusion injury during hepatectomy in HCC patients with chronic HBV infection. World J Surg Oncol 2014;12:27.

104.Li Y, Liu Z, Zhang Y. The effect of intraoperative administration of S-adenosy-methionine on remnant liver function and regeneration after left lobe resection. Chin J Gen Surg 2018;33:955-7.

105. Wen X, Hong Z, Jun C. Liver Function Protective Effect of S-adenosylmethionine on Primary Hepatocellular Carcinoma after Partial Hepatectomy. Shijie Huaren Xiaohua Zazhi 2010;18:3553-8.

106. Fan Y, Wu X. Effect of S-adenosylmethionine on liver protein synthesis and liver function in patients with obstructive jaundice Chin J Gastroent and Hepatol 2014;23:563-5.

107. Guo T, Liu Q. Application value of percutaneous liver puncture biliary drainage and sulfadenosylmethionine before pancreaticoduodenectomy. Chin J Gen Surg 2014;29:965-6.

108. Wang H, Lin N, Wang T, et al. Effect of adenosylmethionine on liver function in patients with hepatolithiasis obstructive jaundice Chin J Prim Med Pharm 2011;18:3357-8.

109. Wang J, Su S, Xu Z. Application of $\mathrm{S}$-adenosylmethionine in the treatment of cholangiocarcinoma with obstructive jaundice. Chin J Modern Drug Application 2011;5:171-2.

110.Zhao L, Pang Y, Liu D. Observation of the effect of biliary stenting combined with ademetionine on malignant obstructive jaundice. Chin J Prim Med Pharm 2013;20:2807-8.

111. Vincenzi B, Daniele S, Frezza AM, et al. The role of $\mathrm{S}$-adenosylmethionine in preventing oxaliplatin-induced liver toxicity: a retrospective analysis in metastatic colorectal cancer patients treated with bevacizumab plus oxaliplatin-based regimen. Support Care Cancer 2012;20:135-9.

112. Vincenzi B, Santini D, Frezza AM, et al. The role of $\mathrm{S}$-adenosyl methionine in preventing FOLFOX-induced liver toxicity: a retrospective analysis in patients affected by resected colorectal cancer treated with adjuvant FOLFOX regimen. Expert Opin Drug Saf 2011;10:345-9.

113. Terrault N, Chen YC, Izumi N, et al. Avatrombopag Before Procedures Reduces Need for Platelet Transfusion in Patients With Chronic Liver Disease and Thrombocytopenia. Gastroenterology 2018;155:705-18.

114.Huang G, Lai EC, Lau WY, et al. Posthepatectomy HBV reactivation in hepatitis B-related hepatocellular carcinoma influences postoperative survival in patients with preoperative low HBV-DNA levels. Ann Surg 2013;257:490-505.

115.Huang G, Lau WY, Wang ZG, et al. Antiviral therapy improves postoperative survival in patients with hepatocellular carcinoma: a randomized controlled trial. Ann Surg 2015;261:56-66.

116.Huang G, Li PP, Lau WY, et al. Antiviral Therapy Reduces Hepatocellular Carcinoma Recurrence in Patients With Low HBV-DNA Levels: A Randomized Controlled Trial. Ann Surg 2018;268:943-54.

117.Infectious Diseases Branch of Chinese Medical Association, Hepatology Branch of Chinese Medical Association. Guidelines for Prevention and Treatment of Chronic Hepatitis B (2019 Edition). Journal of Clinical Hepatobiliary Diseases. 2019;35:2648-69.

118. European Association for the Study of the Liver. Electronic address: easloffice@easloffice.eu; Clinical Practice Guideline Panel: Chair:; Panel members, et al. EASL Clinical Practice Guidelines: Drug-induced liver injury. J Hepatol 2019;70:1222-61.

119. Kulik LM, Atassi B, van Holsbeeck L, et al. Yttrium-90 microspheres (TheraSphere) treatment of unresectable hepatocellular carcinoma: downstaging to resection, RFA and bridge to transplantation. J Surg Oncol 2006;94:572-86.

120.Lewandowski RJ, Kulik LM, Riaz A, et al. A comparative analysis of transarterial downstaging for hepatocellular carcinoma: chemoembolization versus radioembolization. Am J Transplant 2009;9:1920-8.

121. Kaseb AO, Cao HST, Mohamed YI, et al. Final results of a randomized, open label, perioperative phase II study evaluating nivolumab alone or nivolumab plus ipilimumab in patients with resectable HCC. J Clin Oncol 2020;38:abstr 4599. 
122. Yarchoan M, Zhu Q, Durham JN, et al. Feasibility and efficacy of neoadjuvant cabozantinib and nivolumab in patients with borderline resectable or locally advanced hepatocellular carcinoma (HCC). J Clin Oncol 2021;39:abstr 335.

123. Dhir M, Sasson AR. Surgical Management of Liver Metastases From Colorectal Cancer. J Oncol Pract 2016;12:33-9.

124.Zhang W, Hu B, Han J, et al. 174P A real-world study of PD-1 inhibitors combined with TKIs for HCC with major vascular invasion as the conversion therapy: A prospective, non-randomized, open-label cohort study. Ann Oncol 2020;31:S1307.

125. Travis WD, Dacic S, Wistuba I, et al. IASLC Multidisciplinary Recommendations for Pathologic Assessment of Lung Cancer Resection Specimens After Neoadjuvant Therapy. J Thorac Oncol 2020;15:709-40.

126. Edeline J, Boucher E, Rolland Y, et al. Comparison of tumor response by Response Evaluation Criteria in Solid Tumors (RECIST) and modified RECIST in patients treated with sorafenib for hepatocellular carcinoma. Cancer 2012;118:147-56.

127. Wang G, Zhu S, Li X. Comparison of values of CT and MRI imaging in the diagnosis of hepatocellular carcinoma and analysis of prognostic factors. Oncol Lett 2019;17:1184-8.

128. Bertacco A, Vitale A, Mescoli C, et al. Sorafenib treatment has the potential to downstage advanced hepatocellular carcinoma before liver resection. Per Med 2020;17:83-7.

129. Kim TS, Kim JH, Kim BH, et al. Complete response of advanced hepatocellular carcinoma to sorafenib: another case and a comprehensive review. Clin Mol Hepatol 2017;23:340-6.

130.Irtan S, Chopin-Laly X, Ronot M, et al. Complete regression of locally advanced hepatocellular carcinoma induced by sorafenib allowing curative resection. Liver Int 2011;31:740-3.

131. Cleary JM, Tanabe KT, Lauwers GY, et al. Hepatic toxicities associated with the use of preoperative systemic therapy in patients with metastatic colorectal adenocarcinoma to the liver. Oncologist 2009;14:1095-105.

132. Kaseb AO, Pestana RC, Vence LM, et al. Randomized, openlabel, perioperative phase II study evaluating nivolumab alone versus nivolumab plus ipilimumab in patients with resectable HCC. Ann Oncol 2019;37:abstr 185.

133. Kaseb AO, Vence L, Blando J, et al. Immunologic Correlates of Pathologic Complete Response to Preoperative Immunotherapy in Hepatocellular
Carcinoma. Cancer Immunol Res 2019;7:1390-5.

134. Paye F, Jagot P, Vilgrain V, et al. Preoperative chemoembolization of hepatocellular carcinoma: a comparative study. Arch Surg 1998;133:767-72.

135. Li C, Wang MD, Lu L, et al. Preoperative transcatheter arterial chemoembolization for surgical resection of huge hepatocellular carcinoma ( $/=10 \mathrm{~cm})$ : a multicenter propensity matching analysis. Hepatol Int 2019;13:736-47.

136. Majno PE, Adam R, Bismuth H, et al. Influence of preoperative transarterial lipiodol chemoembolization on resection and transplantation for hepatocellular carcinoma in patients with cirrhosis. Ann Surg 1997;226:688-701; discussion 701-3.

137. Tustumi F, Ernani L, Coelho FF, et al. Preoperative strategies to improve resectability for hepatocellular carcinoma: a systematic review and meta-analysis. HPB (Oxford) 2018;20:1109-18.

138.Li N, Feng S, Xue J, et al. Hepatocellular carcinoma with main portal vein tumor thrombus: a comparative study comparing hepatectomy with or without neoadjuvant radiotherapy. HPB (Oxford) 2016;18:549-56.

139. Kudo M. Sequential Therapy for Hepatocellular Carcinoma after Failure of Atezolizumab plus Bevacizumab Combination Therapy. Liver Cancer 2021;10:85-93.

140. Yoo DJ, Kim KM, Jin YJ, et al. Clinical outcome of 251 patients with extrahepatic metastasis at initial diagnosis of hepatocellular carcinoma: does transarterial chemoembolization improve survival in these patients? J Gastroenterol Hepatol 2011;26:145-54.

141.Jung SM, Jang JW, You CR, et al. Role of intrahepatic tumor control in the prognosis of patients with hepatocellular carcinoma and extrahepatic metastases. J Gastroenterol Hepatol 2012;27:684-9.

142.Lee JI, Kim JK, Kim DY, et al. Prognosis of hepatocellular carcinoma patients with extrahepatic metastasis and the controllability of intrahepatic lesions. Clin Exp Metastasis 2014;31:475-82.

143. Uka K, Aikata H, Takaki S, et al. Clinical features and prognosis of patients with extrahepatic metastases from hepatocellular carcinoma. World J Gastroenterol 2007;13:414-20.

144. Bates MJ, Farkas E, Taylor D, et al. Pulmonary resection of metastatic hepatocellular carcinoma after liver transplantation. Ann Thorac Surg 2008;85:412-5.

145. Chen F, Sato K, Fujinaga T, et al. Pulmonary resection for metastases from hepatocellular carcinoma. World J Surg 2008;32:2213-7.

146.Hu Z, Li W, Huang P, et al. Therapeutic significance 
and indications of pulmonary metastasectomy for hepatocellular carcinoma following liver resection. Int J Surg 2017;48:23-31.

147.Lee CY, Bae MK, Park IK, et al. Surgical resection for pulmonary metastasis from hepatocellular carcinoma: analysis of prognosis in relation to primary control. J Surg Oncol 2010;101:239-43.

148. Nakagawa T, Kamiyama T, Nakanishi K, et al. Pulmonary resection for metastases from hepatocellular carcinoma: factors influencing prognosis. J Thorac Cardiovasc Surg 2006;131:1248-54.

149. Nakajima J, Tanaka M, Matsumoto J, et al. Appraisal

Cite this article as: Sun HC, Zhou J, Wang Z, Liu X, Xie Q, Jia W, Zhao M, Bi X, Li G, Bai X, Ji Y, Xu L, Zhu XD, Bai D, Chen Y, Chen Y, Dai C, Guo R, Guo W, Hao C, Huang T, Huang Z, Li D, Li G, Li T, Li X, Li G, Liang X, Liu J, Liu F, Lu S, Lu Z, Lv W, Mao Y, Shao G, Shi Y, Song T, Tan G, Tang Y, Tao K, Wan C, Wang G, Wang L, Wang S, Wen T, Xing B, Xiang B, Yan S, Yang D, Yin G, Yin T, Yin Z, Yu Z, Zhang B, Zhang J, Zhang S, Zhang T, Zhang Y, Zhang Y, Zhang A, Zhao H, Zhou L, Zhang W, Zhu Z, Qin S, Shen F, Cai X, Teng G, Cai J, Chen M, Li Q, Liu L, Wang W, Liang T, Dong J, Chen X, Wang X, Zheng S, Fan J; Alliance of Liver Cancer Conversion Therapy, Committee of Liver Cancer of the Chinese Anti-Cancer Association. Chinese expert consensus on conversion therapy for hepatocellular carcinoma (2021 edition). HepatoBiliary Surg Nutr 2022;11(2):227-252. doi: 10.21037/ hbsn-21-328 of surgical treatment for pulmonary metastasis from hepatocellular carcinoma. World J Surg 2005;29:715-8.

150. Yoon YS, Kim HK, Kim J, et al. Long-term survival and prognostic factors after pulmonary metastasectomy in hepatocellular carcinoma. Ann Surg Oncol 2010;17:2795-801.

151.Kobayashi S, Takahashi S, Kato Y, et al. Surgical treatment of lymph node metastases from hepatocellular carcinoma. J Hepatobiliary Pancreat Sci 2011;18:559-66.

152.Park JS, Yoon DS, Kim KS, et al. What is the best treatment modality for adrenal metastasis from hepatocellular carcinoma? J Surg Oncol 2007;96:32-6. 
Supplementary

Table S1 Asia clinical guidelines, clinical consensus statements, and systematic reviews on treatment of HCC

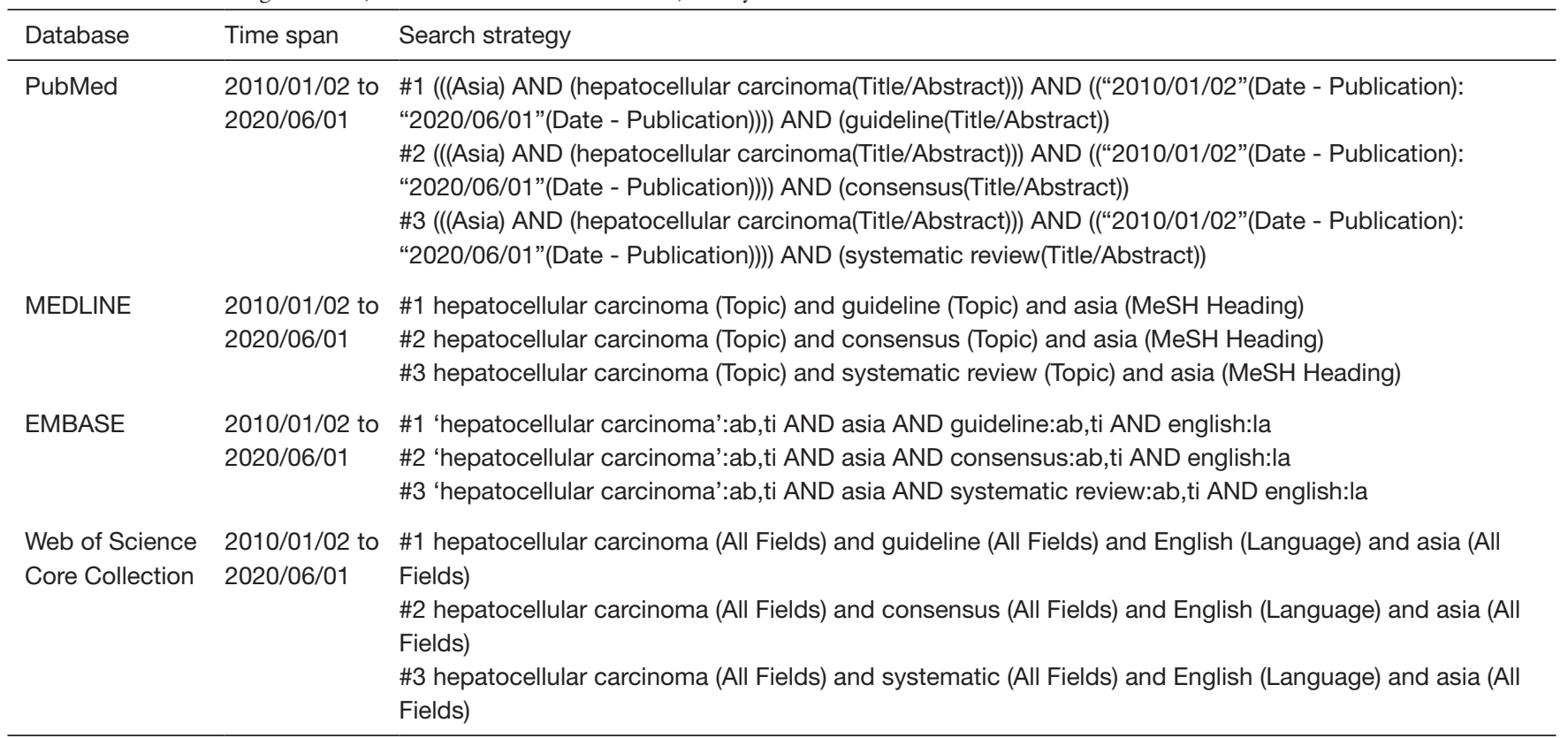

Table S2 Hepatocellular carcinoma \& conversion therapy/downstaging

\begin{tabular}{|c|c|c|}
\hline Database & Time span & Search strategy \\
\hline MEDLINE & $\begin{array}{l}1950-01-01 \text { to } \\
2020-08-01\end{array}$ & $\begin{array}{l}\text { \#1 advanced hepatocellular carcinoma (Topic) } \\
\text { \#2 initial unresectable hepatocellular carcinoma (Topic) } \\
\text { \#3 conversion therapy (Topic) } \\
\text { \#4 downstaging (Topic) } \\
\text { \#5 (\#1) AND (\#3) } \\
\text { \#6 (\#2) AND (\#3) } \\
\text { \#7 (\#1) AND (\#4) } \\
\text { \#8 (\#2) AND (\#4) }\end{array}$ \\
\hline $\begin{array}{l}\text { Web of Science } \\
\text { Core Collection }\end{array}$ & & $\begin{array}{l}\text { \#1 TS=(hepatocellular carcinoma* conversion therapy) } \\
\text { \#2 TS=(hepatocellular carcinoma* downstaging) } \\
\text { \#3 TS=(initially unresectable hepatocellular carcinoma* surgery) } \\
\text { \#4 TS=(initially unresectable hepatocellular carcinoma* resectable) }\end{array}$ \\
\hline $\begin{array}{l}\text { Search trials } \\
\text { (CENTRAL) in } \\
\text { Cochrane Library }\end{array}$ & & $\begin{array}{l}\# 1 \text { "hepatocellular carcinoma" in All Text AND conversion therapy in Title Abstract Keyword OR } \\
\text { downstaging in Title Abstract Keyword } \\
\# 2 \text { "initially unresectable hepatocellular carcinoma" in All Text AND surgery in Title Abstract Keyword OR } \\
\text { resectable in Title Abstract Keyword }\end{array}$ \\
\hline
\end{tabular}


Table S3 The discipline and geographical distribution of respondents

\begin{tabular}{|c|c|}
\hline Name & Affiliation \\
\hline Weidong Jia & Department of Liver Surgery, The First Affiliated Hospital of USTC, Hefei, China \\
\hline Xinyu Bi & $\begin{array}{l}\text { Department of Hepatobiliary Surgery, National Cancer Center/National Clinical Research Center for Cancer/Cancer } \\
\text { Hospital, Chinese Academy of Medical Sciences and Peking Union Medical College, Beijing, China }\end{array}$ \\
\hline Gong Li & Department of Radiation Oncology, Beijing Tsinghua Changgung Hospital, Beijing 102218, China \\
\hline Xueli Bai & $\begin{array}{l}\text { Department of Hepatobiliary and Pancreatic Surgery, the First Affiliated Hospital, School of Medicine, Zhejiang University, } \\
\text { Hangzhou } 310003 \text {, China }\end{array}$ \\
\hline $\mathrm{Li} \mathrm{Xu}$ & Department of Liver Surgery, Sun Yat-sen University Cancer Centre, Guangzhou, China \\
\hline Zheng Wang & $\begin{array}{l}\text { Department of Liver Surgery, Liver Cancer Institute, Zhongshan Hospital, Fudan University, Key Laboratory of } \\
\text { Carcinogenesis and Cancer Invasion, Fudan University, Ministry of Education, Shanghai 200032, China }\end{array}$ \\
\hline Xiaodong Zhu & $\begin{array}{l}\text { Department of Liver Surgery and Transplantation, Liver Cancer Institute, Zhongshan Hospital, Fudan University, Shanghai, } \\
\text { China }\end{array}$ \\
\hline Bixiang Zhang & $\begin{array}{l}\text { Hepatic Surgery Center, Tongji Hospital, Tongji Medical College, Huazhong University of Science and Technology, } 1095 \\
\text { Jiefang Avenue, Wuhan 430030, China }\end{array}$ \\
\hline Dousheng Bai & Department of Hepatobiliary Surgery, Clinical Medical College, Yangzhou University, Yangzhou, China \\
\hline Yajin Chen & Department of Hepatobiliopancreatic Surgery, Sun Yat-sen Memorial Hospital, Sun Yat-sen University, Guangzhou, China. \\
\hline Wenzhi Guo & $\begin{array}{l}\text { Department of Hepatobiliary and Pancreatic Surgery, The First Affiliated Hospital of Zhengzhou University, Zhengzhou } \\
\text { 450052, China }\end{array}$ \\
\hline Chunyi Hao & $\begin{array}{l}\text { Key Laboratory of Carcinogenesis and Translational Research (Ministry of Education), Sarcoma Center, Peking University } \\
\text { Cancer Hospital and Institute, Beijing, China }\end{array}$ \\
\hline Tao Huang & Department of Hepatobiliary Surgery, Affiliated Tumour Hospital of Zhengzhou University, Zhengzhou 450008, China \\
\hline Zhiyong Huang & $\begin{array}{l}\text { Hepatic Surgery Center, Tongji Hospital, Tongji Medical College, Huazhong University of Science and Technology, Wuhan, } \\
\text { China }\end{array}$ \\
\hline Deyu Li & Department of Hepato-Biliary Pancreatic Surgery, Henan Provincial People’s Hospital, Zhengzhou, China \\
\hline Tao Li & Department of general surgery, Qilu Hospital, Shandong University, Jinan 250012, China \\
\hline Xiangcheng Li & $\begin{array}{l}\text { Department of Liver Transplantation Center, The First Affiliated Hospital of Nanjing Medical University, Nanjing, Jiangsu, } \\
\text { 201129, China }\end{array}$ \\
\hline
\end{tabular}

Table S3 (continued) 
Table S3 (continued)

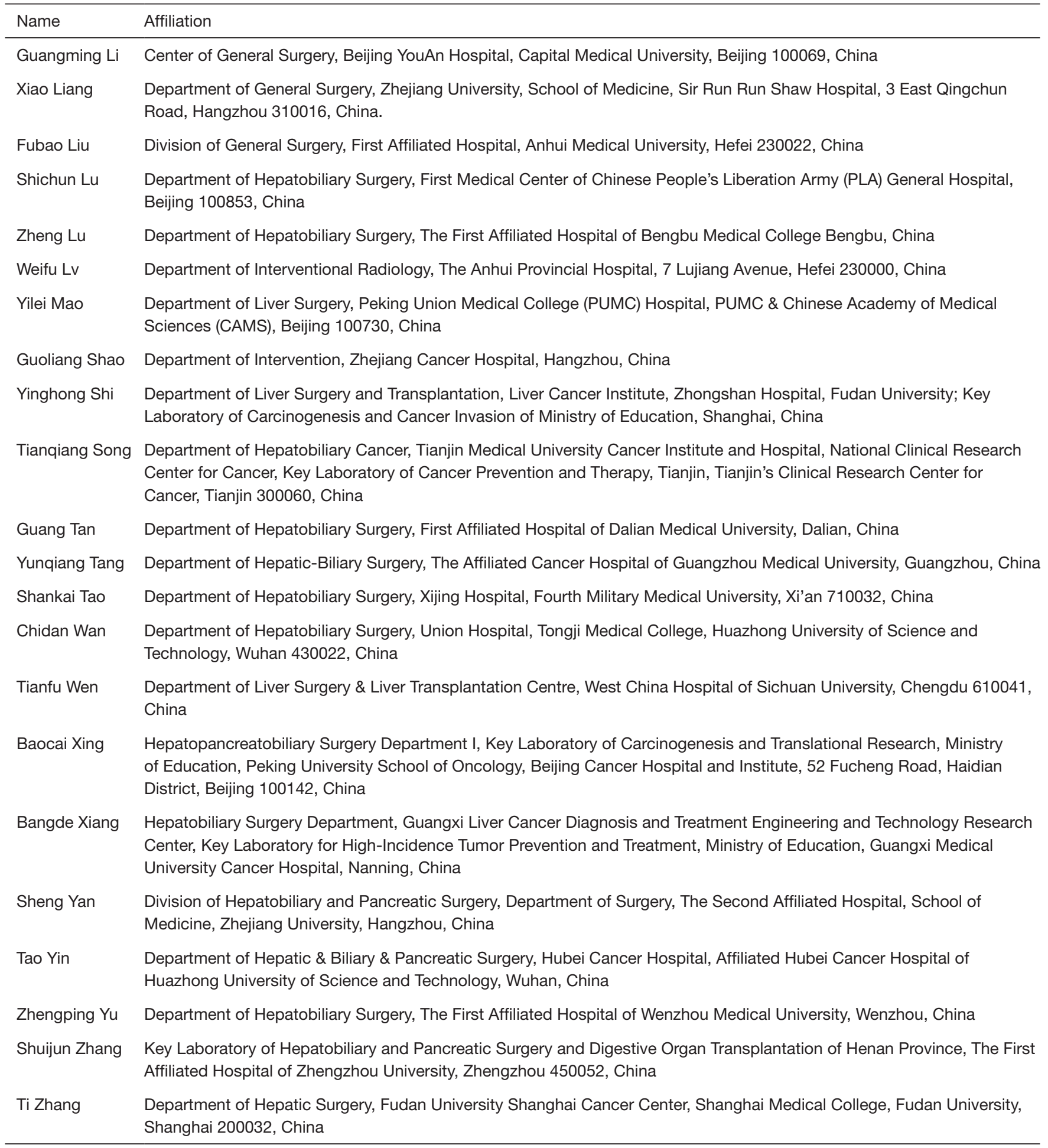

Table S3 (continued) 
Table S3 (continued)

\begin{tabular}{|c|c|}
\hline Name & Affiliation \\
\hline Aibin Zhang & $\begin{array}{l}\text { Department of Hepatobiliary Pancreatic Surgery, The First Affiliated Hospital of College of Medicine, Zhejiang University, } \\
\text { Hangzhou } 310003 \text {, China }\end{array}$ \\
\hline Ledu Zhou & Department of Liver Surgery, Xiangya Hospital, Central South University, Changsha, China \\
\hline Zhenyu Zhu & Hepatoliliary Surgery Center, 302 Hospital of PLA, Beijing, China \\
\hline
\end{tabular}

